\title{
B. Regionale Strukturen
}

\section{Postindustrielle wirtschaftsgeographische Strukturen}

In nur rund 100 Jahren vollzog sich die Entwicklung der USA von einer Agrarnation zur führenden Industriemacht bis hin zur "postindustriellen" Dienstleistungsgesellschaft, deren Ende bereits vorausgesagt wird (Thurow 1989). Diese Wirtschaftsentwicklung hat das Bild Amerikas entscheidend verändert. So wie sich im Industriezeitalter ein zentral-peripheres wirtschaftsgeographisches Gefüge herausbildete, in dem der manufacturing belt das wirtschaftliche "Zentrum" und der Rest der USA die strukturschwache "Peripherie" (vgl. dazu Schätzl 1981: 123) darstellten, so kennzeichnet die postindustrielle Ära seit ungefähr 1950 eine neue Wirtschaftsgeographie (vgl. Sternlieb/Hughes 1978). Ihre wichtigsten Merkmale sind:

(1) eine industrielle Mehrkernstruktur, d.h. die Ausbildung neuer Industrieregionen außerhalb des klassischen "altindustrialisierten" manufacturing belt,

(2) der Aufschwung des tertiären Sektors in alt- und neuindustrialisierten städtischen Ballungsräumen, sowie aufgrund staatlicher Maßnahmen in einigen ländlichen, dünner besiedelten, strukturschwachen Regionen und

(3) im Agrarsektor regional verschiedene Anpassungen an die "Farmkrise" durch Änderung der Betriebsgrößen, Organisationsformen und regionale Verlagerung von Produktionsschwerpunkten.

Ziel des vorliegenden Beitrags ist es, diese regionalen Strukturen der USWirtschaft mit Ausnahme von Alaska, Hawaii und anderer US-Territorien aufgrund der Literatur und neuester Statistiken zusammenzufassen. Schwerpunktmäßig behandelt werden:

(1) die Ausbildung des manufacturing belt zum wirtschaftlichen Zentrum der USA im Industriezeitalter sowie der postindustrielle Wandel zu einer industriellen Mehrkernstruktur,

(2) die Tertiarisierung der US-Wirtschaft sowie die geographische Verteilung und Konzentration ausgewählter Dienstleistungsbereiche und

(3) postindustrielle sektorale und regionale Strukturen in der Landwirtschaft.

Diese Thematik ist nicht neu, da es ein Grundgedanke der Wirtschaftsgeographie ist, daß die jeweilig feststellbaren geographischen Verteilungsmuster nicht endgültig sind,

Geographical distributions are brought about by processes that are an integral part of the economy and society, indeed they reflect and 
maintain these processes. ... as social and economic development proceeds, so the social formation changes and, in consequence new and different geographical patterns and distributions are created (Clark 1985: 3).

Generationen von Wirtschaftsgeographen und anderen Sozialwissenschaftlern befaßten sich daher mit der regionalen Ausbildung von Industrie, Gewerbe und Landwirtschaft, der Verlagerung von Standorten, dem Ausbau oder dem Abbau von Arbeitsplätzen in Räumen, die von der Infrastruktur erschlossen oder vom technischen Fortschritt überholt wurden. Die regionalen Schwerpunkte von Industrie, Dienstleistungen und der Landwirtschaft sind in jüngster Zeit von einer großen Anzahl von Autoren behandelt worden (s. Literaturverzeichnis, wovon Birdsall/Florin (1981), Clark (1985), Malecki (1980, $1981,1984,1985)$ und Windhorst $(1986,1987)$ für das vorliegendc Kapitcl eine besonders wichtige Grundlage bilden).

Im folgenden werden alte und neue Verteilungsmuster des Industriesektors angesprochen. Dabei, wie auch bei der Betrachtung des Dienstleistungsund Agrarsektors wird versucht, die wirtschaftliche, politische und soziale Gesamtsituation in die spezifisch geographische, raumbezogene Betrachtungsweise einzubeziehen.

\section{Der Industriesektor}

\subsection{Der manufacturing belt als wirtschaftliches Kernland}

Die rasche Entwicklung der USA von einer Agrarnation zur führenden Industriemacht wurde durch das Bevölkerungswachstum beschleunigt. Während 1820 9,6 Mio. Menschen im Census erfaßt wurden, waren es 1920 schon 105,7 Mio., 1970 rund 203,3 Mio. und 1980 über 226,5 Mio.; allein durch Einwanderung stieg die Bevölkerung zwischen 1820 und 1988 um 54,4 Mio. an (U.S. Bureau of the Census 1990: 7,9). Der Bevölkerungsanstieg garantierte eine große Nachfrage und sichere Absatzmärkte für die Wirtschaft und ermöglichte so die Industrialisierung, die sich später aufgrund von staatlicher Protektion eine führende Stellung auf dem Binnenmarkt sowie auf dem Weltmarkt aufbauen konnte (Holtfrerich 1987). Frühes industrielles Wachstum und Ausbildung des Handels waren zuerst in den Metropolen der Ostküste zu verzeichnen, weil dort das größte Bevölkerungswachstum stattfand. Es ist die Gegenküste zu Europa, der Großteil der Einwanderer landete dort, brachte Arbeitskraft, Erfahrung und Ideen. Die weitere Industrialisierung im Hinterland der Ostküste bis in den Mittelwesten wurde durch sukzessive Westwärtswanderungen der Bevölkerung und punktuelle Erschließung der Ressourcen (industrial nucleation) eingeleitet (Perloff u.a. 1960). Wichtig dabei war auch die symbiotische Beziehung der neuen Industrien mit der Landwirtschaft:

... success in agriculture supported the region's early market centers, but it was the gradual mechanization of agriculture that de- 
manded diversified manufacturing support. Mechanical reapers, winnowing machines and cultivating implements by the tens of thousands were required even before the turn of the century... increasingly specialized farm machinery continued to be important local sources of industrial demand during the first half of this century. And transportation lines were improved and expanded to carry the tremendous volume of agricultural products that were produced on the region's farms. Thus, agriculture stimulated early industrial and urban growth and successful manufacturing and rapidly growing urban centers continued intensification and improvement in agriculture (Birdsall/Florin 1981: 93).

Es entstand die duale Wirtschaftsstruktur des amerikanischen Nordostens, der neben dem manufacturing belt von jeher wesentliche Teile des landwirtschaftlichen Kernlandes der USA einschloß (Birdsall/Florin 1981: 93 und Schaubilder B-1 und B-2).

Schaubild B-1: Der manufacturing belt

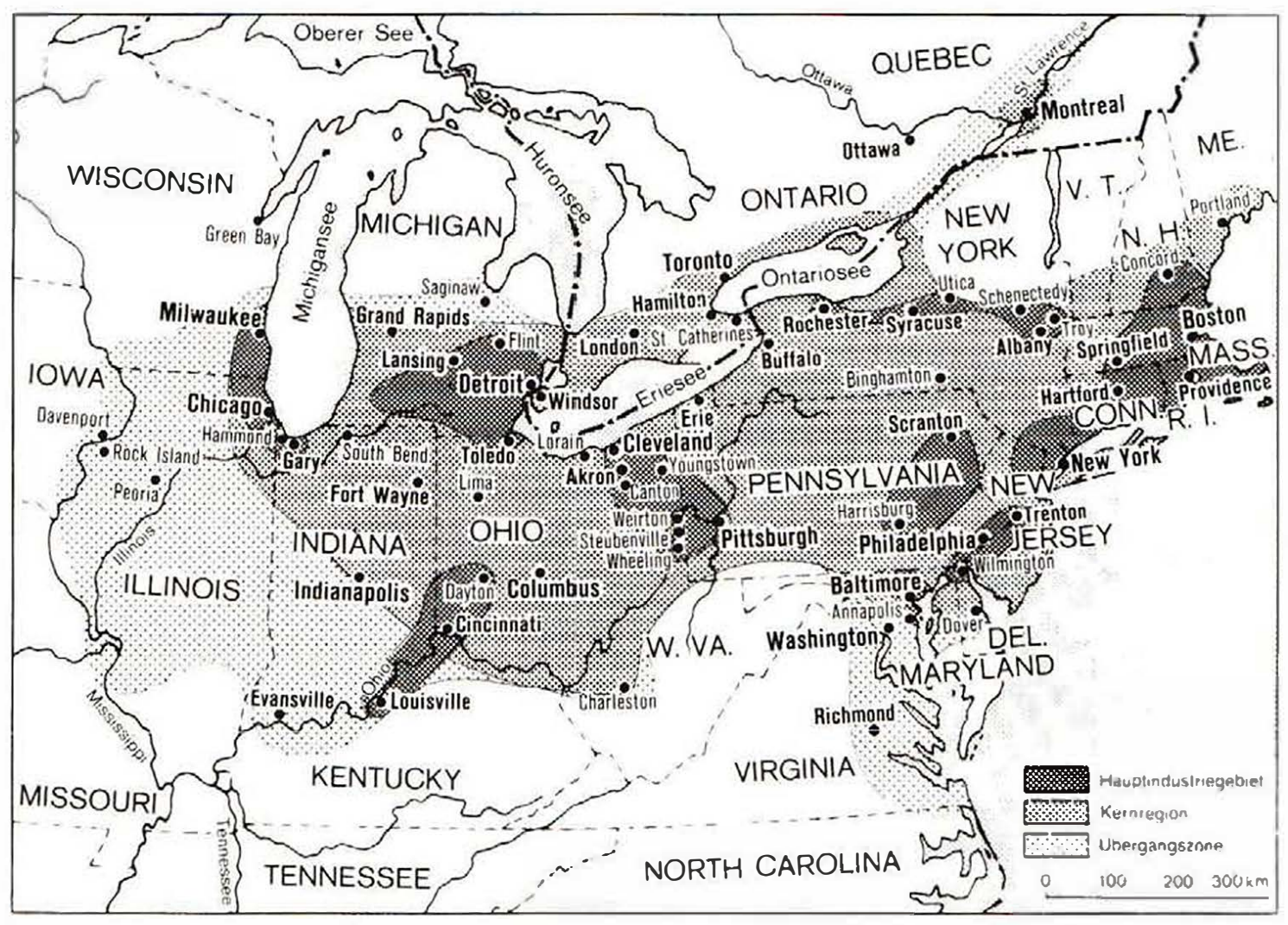

Quelle: Nach Birdsall/Florin 1981: 92; Clark 1985: 82.

Die industrielle Ent wicklung fand also nicht in einem ununterbrochenen Gürtel, sondern in wenigen Teilgebieten statt (vgl. Schaubild B-1), die sich durch ihre Häfen, allgemeine Verkehrsgunst oder Ressourcen auszeichneten, jedoch hinsichtlich ihrer Spezialisierung und Anpassungsfähigkeit an neue wirtschaftliche Gegebenheiten erhebliche Unterschiede aufwiesen (Clark 1985: 8192). $\mathrm{Zu}$ den regionalen Standortschwerpunkten gehören die Industrieagglome- 
rationen an der atlantischen Küste, vor allem die Großräume Boston, New York/New Jersey, Philadelphia und Baltimore, die schon vor der Industrialisierung als dichtbesiedelte Räume und wegen ihrer Häfen eine gute Grundlage für Handel und Gewerbe boten, sowie die Industriezentren im Innern des Landes zwischen dem Ohio River und den südlichen Großen Seen. Die Entwicklung dieser Teilräume ist ausführlich in Birdsall/Florin (1981), Clark (1985) und Blume (1987, Bd. 1) dargestellt, so daß hier nur ein kurzer Abriß der heutigen Struktur erfolgt.

Schaubild B-2: Die landwirtschaftliche Kernregion der USA

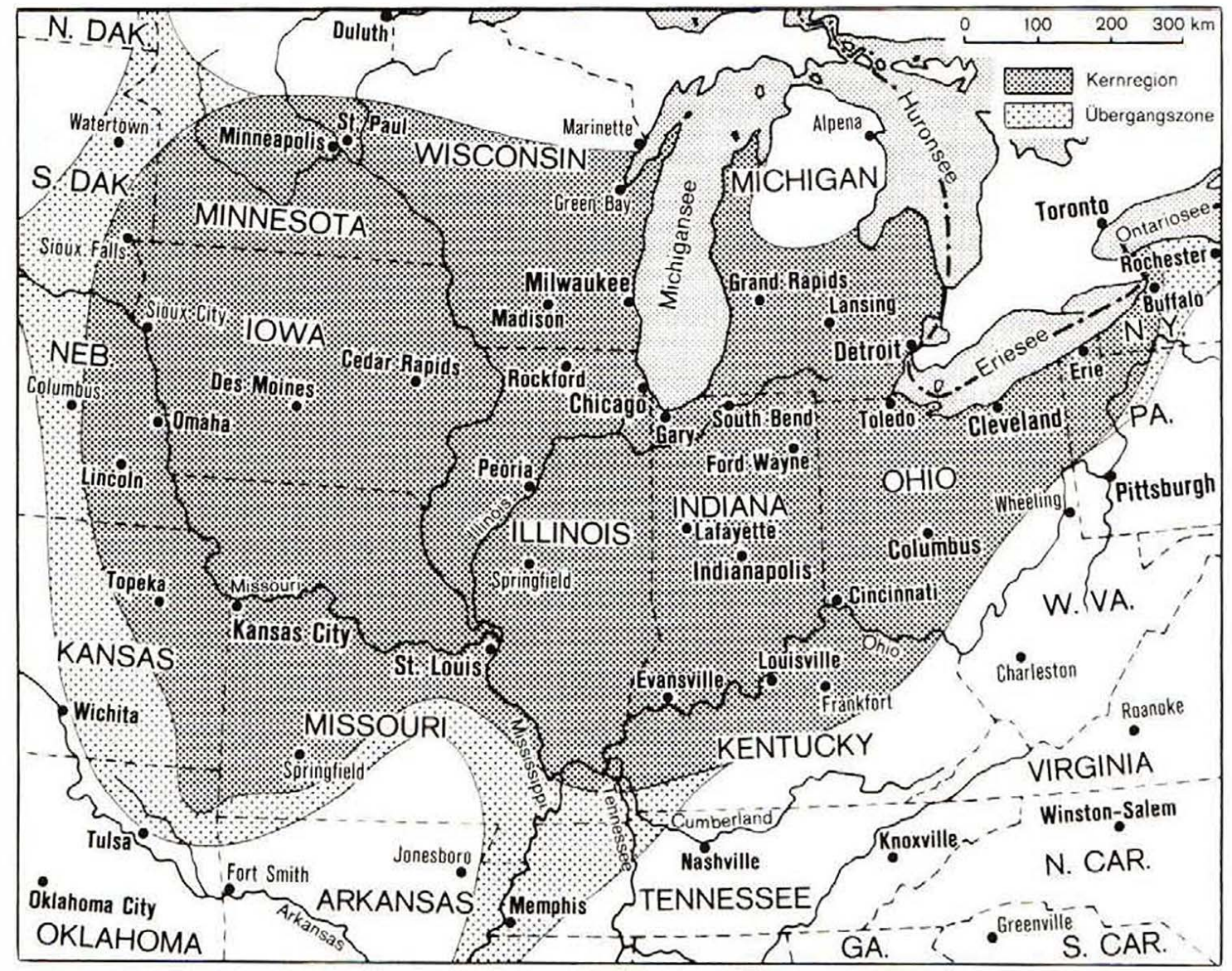

Quelle: Birdsall/Florin 1981: 270.

Im südlichen Neuengland wurde nach dem Rückgang traditioneller Industrien, wie z.B. Metallverarbeitung, Maschinenbau, Holzwirtschaft und Textilindustrie, das Arbeitsplatzwachstum in neuartigen Industrien angeregt (Breuer 1984). Seit den 50er Jahren gibt es im Raum Boston, vor allem entlang des electronics highway (Route 128) eine starke Konzentration der Elektronikund Hochtechnologiebranchen, die durch die Forschungseinrichtungen und bedeutenden Universitäten angezogen wurden, z.T. sogar deren spin-offs sind (Hall/Markusen 1985). Der Großraum New York ist zwar nach Anzahl und Vielfalt der Industriebetriebe der bedeutendste Industriestandort im manufacturing belt, hat aber in der Beschäftigung einen Schwerpunkt auf Textil- und 
Bekleidungsindustrie sowie Verlags- und Druckereiwesen. Seit den 70er Jahren wurden ferner mit gutem Erfolg Anreize für die wachstumversprechende Computer- und Elektronikindustrie geschaffen; die Stadtlandschaft ist heute weitgehend tertiärwirtschaftlich, d.h. von den office industries, wie z.B. Konzernverwaltungen, Banken und Versicherungen von internationaler Bedeutung, geprägt (Birdsall/Florin 1981: 105). Die Großräume New Jersey, Philadelphia und Baltimore weisen auch in den 80er Jahren trotz wiederholter konjunktureller Einbußen bedeutende Konzentrationen der petrochemischen Industrie, Eisen- und Stahlverarbeitung, sowie des Maschinen-, Werkzeug- und Schiffsbaus auf. Hinsichtlich der letztgenannten Industriezweige erfuhren diese Standorte auf der Grundlage von billigen importierten Erzen gerade in der postindustriellen Zeit einen wichtigen Aufschwung. Preispolitik, wie z.B. das Basing Point Price System und sein Vorläufer, das Pittsburgh Plus System hatten vorher Standortverlagerungen der Eisen- und Stahlindustrie an die Ost-, Westund Südküste erschwert (Clark 1985: 89). Der Großraum Pittsburgh, in dem man seit Mitte der 40er Jahre gezielt versuchte, eine neue wirtschaftliche Basis zu schaffen, gilt seit Mitte der 80er Jahre nicht länger als smoke city sondern als software city (Deysson 1988; Economou 1987), wie weiter unten dargelegt wird.

Die Stadträume Chicago-Gary und Detroit, die aufgrund von Eisenerz und Kohle eine bedeutende Stahlproduktion und als deren Hauptabnehmer die Automobilindustrie ausgebildet hatten, konnten ihre Bedeutung in der postindustriellen Zeit weiter ausbauen bzw. trotz Konjunkturschwankungen aufrechterhalten (Friese/Hofmeister 1983: 72). Im Gegensatz zu Detroit aber war Chicagos wirtschaftliche Basis sehr früh diversifiziert und weist heute u.a. eine bedeutende Elektroindustrie, Apparatebau, Maschinenbau und Metallverarbeitung, chemische Industrie, Möbel- und Lebensmittelproduktion, Druckerei- und Verlagswesen auf. Diversifiziert sind auch die Industrieregionen in Süd-West Ohio, Süd-Ost Indiana und angrenzenden Gebiete in Kentucky, die neben Eisen- und Stahlindustrie den Bau von Maschinen, Werkzeugen, Computern, Flugzeug- und Raketenteilen sowie von anderen strategisch wichtigen Produkten aufweisen und daher ganz wesentlich durch Regierungsaufträge gestärkt werden (Clark 1985; U.S. Department of Defense 1985). Die Industriestädte des Mohawk Valley haben mit Textilindustrie, Metallverarbeitung und Maschinenbau, Elektronik- und Optikindustrie eine breite wirtschaftliche Grundlage geschaffen; die Städte zwischen Pittsburgh und Cleveland sind dagegen einseitiger auf Stahl und Automobilteile sowie Industrie der Steine und Erden ausgerichtet (Clark 1985: 87-88).

\subsection{Gegenwärtige Bedeutung des manufacturing belt}

Der manufacturing belt hat mit der Entwicklung der USA zu einer Dienstleistungsgesellschaft einen allgemeinen und in den 70er Jahren besonders starken Rückgang hinsichtlich der Bevölkerung und der Arbeitsplätze erfahren (Voll- 
mar/Hopf 1987: 468; Clark 1985: 100-107; Crandall 1988). Trotz des Rückgangs bleibt der manufacturing belt aber auch in den 80er Jahren nicht nur die bedeutendste Industrieregion der USA, sondern erfährt teilweise sogar einen wirtschaftlichen Aufschwung, der sich in stark sinkenden Arbeitslosenquoten, Arbeitsplatzwachstum bei Hochtechnologieindustrien und ausgewählten Dienstleistungen sowie im z.T. stark steigenden Realeinkommen manifestiert (Schneider-Sliwa 1989). Tabelle B-1 zeigt, daß die Regionen (im Census als geographic divisions bezeichnet), die den manufacturing belt ausmachen - das sind New England, East North Central und Middle Atlantic (vgl. Schaubild B-3) - 1977 zusammen 51,0\% (10 Mio.) und 1986 45,9\% (8,4 Mio.) der industriellen Arbeitsplätze sowie 1977 insgesamt $51,1 \%$ und $198646,4 \%$ der industriellen Wertschöpfung der USA auf sich vereinten; Schwerpunkte liegen dabei auf der Metallverarbeitung, dem Maschinen- und Werkzeugbau, dem Fahrzeug-, Flugzeugteile-, Schiffs- und Eisenbahnbau, der Raumfahrtausrüstung sowie der Elektronik und Elektrotechnik.

Tabelle B-1: Regionale Anteile an den Beschäftigten und an der Wertschöpfung in der Industrie (in \%)

\begin{tabular}{lccrr}
\hline \multirow{2}{*}{ Regiona } & \multicolumn{2}{c}{ Beschäftigte } & \multicolumn{2}{c}{ Wertschöpfung } \\
& 1977 & 1986 & 1977 & 1986 \\
& $(19,59$ Mio. $)$ & $(18,37$ Mio. $)$ & & \\
\hline New England & 7,1 & 7,4 & 6,1 & 6,9 \\
Middle Atlantic & 18,5 & 16,3 & 17,6 & 15,8 \\
East North Central & 25,4 & 22,2 & 27,4 & 23,7 \\
West North Central & 6,6 & 6,9 & 6,9 & 7,4 \\
South Atlantic & 14,4 & 16,2 & 12,4 & 15,1 \\
East South Central & 6,8 & 6,7 & 6,2 & 6,2 \\
West South Central & 7,4 & 7,7 & 8,9 & 8,4 \\
Mountain & 2,4 & 3,1 & 2,3 & 3,1 \\
Pacific (ohne Alaska & 11,3 & 13,3 & 12,0 & 13,4 \\
und Hawaii) & & & & \\
\hline
\end{tabular}

Quelle: Nach U.S. Bureau of the Census 1989: 726, Tab. 1268 (veränd., eig. Berechnung).

a Region bezeichnet hier, in den folgenden Tabellen sowie im Text die in Schaubild B-3 dargestellten geographic divisions des Census.

\subsection{Industriezentren außerhalb des manufacturing belt}

Die Herausbildung wichtiger Industriezentren außerhalb des manufacturing belt in den vergangenen 40 Jahren war von mehreren Faktoren beeinflußt (Berentsen 1987: 465; Friese/Hofmeister 1983: 73), vor allem: 


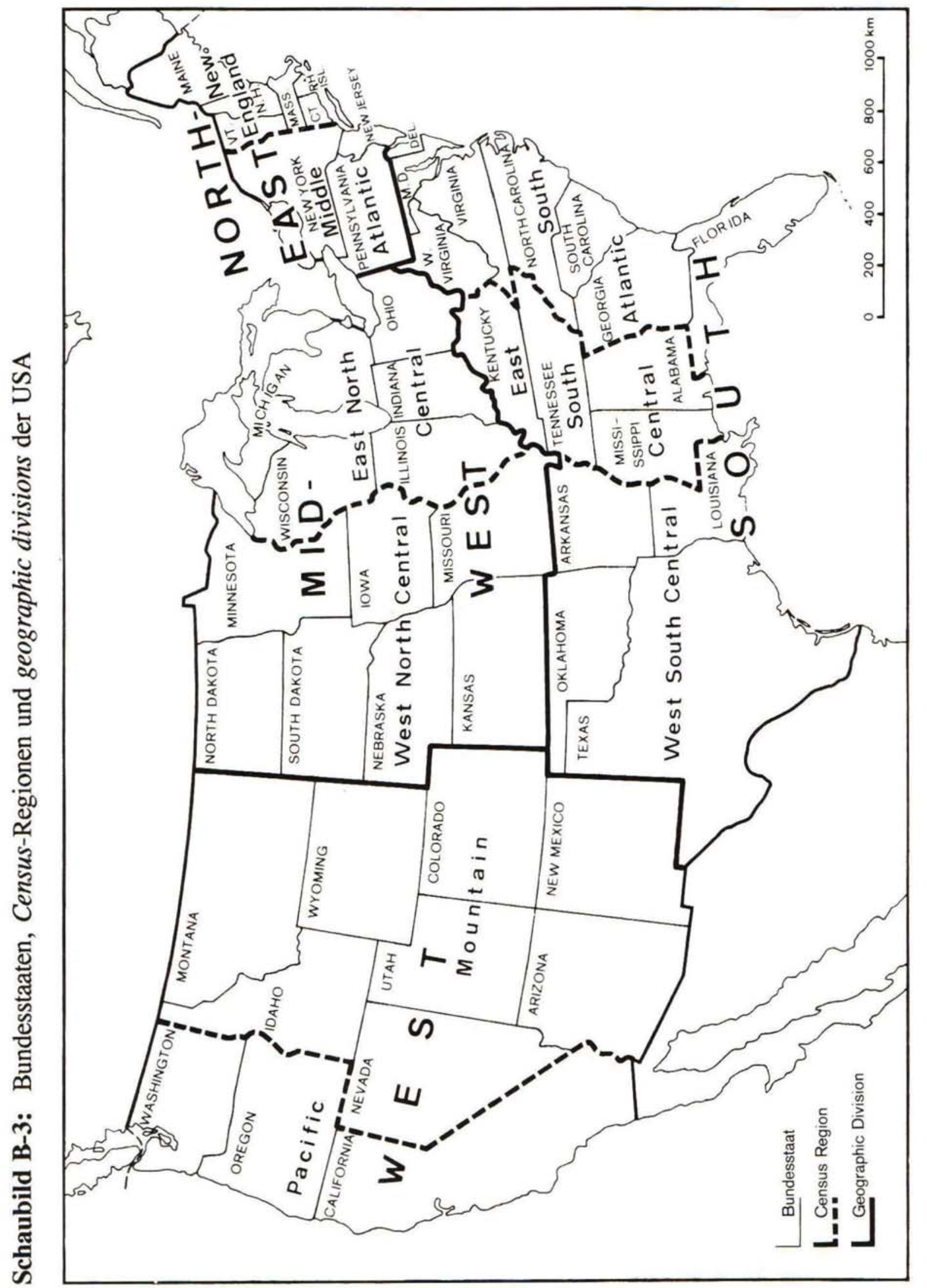

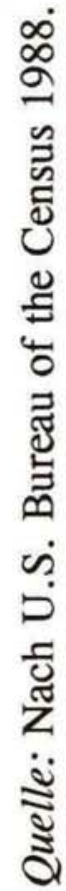


(1) Verkehrsverbesserungen im Süden und Westen, z.B. dem Ausbau des Highway-Systems und nachfolgender Ansiedlung von Industriebetrieben,

(2) Nutzung neuer Energiequellen, die zu einem Bedeutungsverlust des Standortfaktors Kohle führte,

(3) Mechanisierung der Agrarwirtschaft mit Freisetzung von billigen und gewerkschaftlich weniger organisierten Arbeitskräften,

(4) Subventionen und Regierungsaufträgen in Gebieten mit freundlicherem Geschäftsklima (niedrigen Löhnen, Steuervorteilen, geringerer Gewerkschaftsmitgliedschaft),

(5) ökologischer Überbelastung des manufacturing belt, erhöhtem Stellenwert des Klimas und der Natur, was die Migrationsbereitschaft der Erwerbstätigen und die Verlagerung von Spezialindustrien begünstigte.

Aufgrund dieser Faktoren entstand im Pazifischen Nordwesten, im Siidwesten und im Süden seit dem Zweiten Weltkrieg ein wirtschaftliches, politisches und kulturelles Gegengewicht zum industriellen Kernland. Dies wurde durch Investitionen in Branchen erreicht, die seither zu den "basic pillars of the cowboy economy" wurden, z.B. Rüstungsindustrie, Flugzeugbau und Weltraumtechnik, Elektronik, Öl- und Erdgasförderung, petrochemische Industrie, Tourismus sowie Agrarwirtschaft (Vollmar/Hopf 1987: 468; Sale 1975).

Infolge des verbesserten, unternehmerfreundlichen Klimas im subventionierten, ökologisch sauberen pazifischen Nordwesten und dem sunbelt verzeichneten diese Regionen vor allem in den 70er Jahren ein starkes prozentuales Wachstum der Industriebeschäftigten, der Nordosten dagegen einen Rückgang. In den 80er Jahren allerdings zeigen sich auch im sunbelt Stagnation und Einbußen im Beschäftigungsbereich (z.B. Texas, vgl. Schaubild B-4; Sternlieb 1986; Schneider-Sliwa 1989).

Betrachtet man die postindustrielle Entwicklung des sunbelt, so fallen drei Teilstücke auf, die sich hinsichtlich der Art der Industrialisierung, des Lohnniveaus und der Stabilität des Wachstums unterscheiden: Der Golfküstenraum westlich des Mississippi, der "Alte Süden" und Kalifornien. Die industrielle Entwicklung des Golfküstenraums ist eng mit direkten und indirekten staatlichen Maßnahmen verbunden, z.B. Steuererleichterungen oder der Verlagerung von Produktionsstätten im Zweiten Weltkrieg, der Verkehrserschließung durch den Küstenwasserweg, der eine Verbindung über den Mississippi und den Ohio zu den Großen Seen ermöglicht. Trotz der Fördermaßnahmen der öffentlichen Hand, bei der auch einzelstaatliche und lokale Verwaltungsebenen eine Rolle spielen, ist diese Region, die hinsichtlich der Branchen, der Produktivität und der Lohnraten in den siebziger Jahren ein starkes Wachstum zeigte, mit der Problematik einer monostrukturierten Wirtschaft belastet, wie sich zu Anfang der 80er Jahre zeigte (Friese/Hofmeister 1983: 75; Blume 1987, Bd. 1 und Schaubild B-4). 


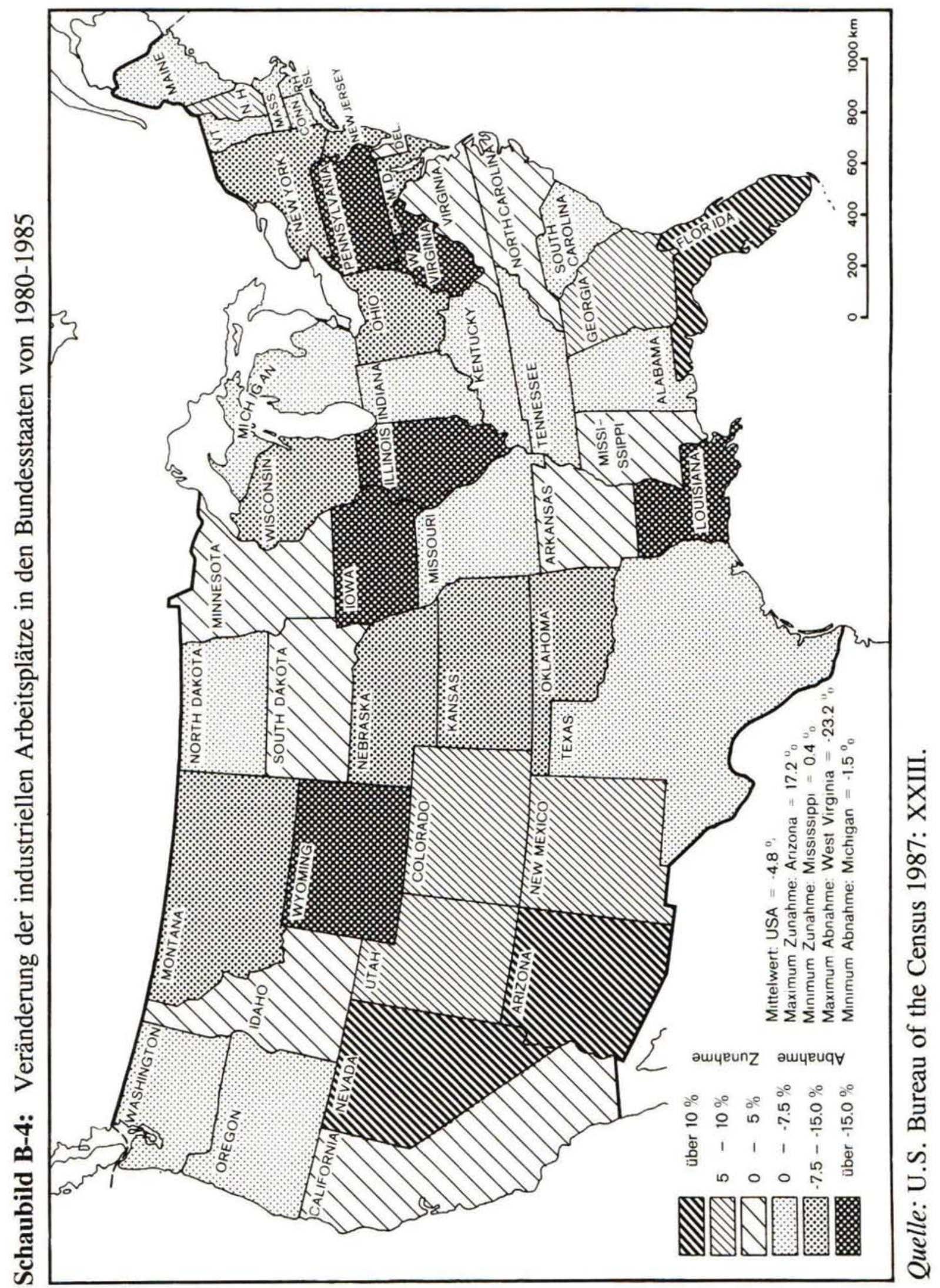


Teile des Alten Südens zeigen in den 70er und 80er Jahren ein markantes Wachstum industrieller Arbeitsplätze (vgl. Schaubild B-4), das sich vor allem im nichtmetropolitanen Raum in vielen Kleinbetrieben vollzieht, die hinsichtlich ihrer Produktionsfaktoren nicht standortgebunden sind, wie z.B. Lebensmittelproduktion, Textil- und Bekleidungsindustrie, Holz- und Möbelverarbeitung sowie die Elektroindustrie. Es ist weniger die staatliche Förderung als die Verfügbarkeit billiger Arbeitskräfte, die das Aufkommen dieser Industrien im ländlichen Bereich erklärt, denn für relativ arbeitsintensive Betriebe mit hohem Bedarf an ungelernten oder angelernten Arbeitskräften sind die Voraussetzungen dort günstiger (Birdsall/Florin 1981: 237). Das niedrige Lohnniveau im Südosten erklärt sich z.T. aus der traditionell mangelhaften gewerkschaftlichen Organisierung der Arbeitnehmerschaft, die in jüngster Zeit noch abnimmt (vgl. Schaubild B-5 und das Kapitel $L$. Gewerkschaftsbewegung und Arbeitsbeziehungen in diesem Buch). Für die Ansiedlung der Stahlindustrie ("mini-mills") war dies ein besonders wichtiger Faktor (Scherrer 1987).

Hinsichtlich des Wohlstands ist der Alte Süden trotz der neuen Industrialisierung auch in den 80er Jahren immer noch eine der ärmsten Regionen der USA; das Pro-Kopf-Einkommen liegt in fast allen Staaten unter dem nationalen Durchschnitt (vgl. Schaubild B-6), es ist sogar eine neue Nord-Süd-Polarisierung hinsichtlich der Realeinkommen festzustellen (Schneider-Sliwa 1989), und der Anteil der Sozialhilfeempfänger im Süden liegt über dem nationalen Mittelwert (vgl. Schaubild B-7). Auch in den achtziger Jahren gilt die folgende Aussage über den Süden:

... the rural South's attractiveness seems to lie largely in its pool of underemployed and relatively unskilled labor, the growth of laborintensive and low-wage sectors will not bring the incomes of rural Southerners to the level of the nation as a whole (Hansen 1974, zit. bei Birdsall/Florin 1981: 40).

Die Arbeitslosenquoten (für die civilian, non-institutionalized Bevölkerung) lagen in Teilen des Alten Südens 1986 noch weit über dem nationalen Mittelwert von 7,0\% oder stiegen an, obwohl sie zwischen 1980 und 1986 (vor allem ab 1982/83) in fast allen übrigen Bundesstaaten sanken (Schaubild B-8). Dies weist auf die hohe strukturelle Arbeitslosigkeit der Region hin. Kalifornien, das schon seit Beginn des 20. Jahrhunderts Erdöl-, Rüstungsindustrie und Flugzeugbau aufwies, erhielt in der postindustriellen Ära einen starken Impuls durch die Konzentration von Hochtechnologiefirmen im Silicon Valley südlich von San Francisco. Dort wuchs die Beschäftigtenzahl zwischen 1949 und 1980 um 1000\% (von rund 60.000 auf ca. 652.000) in den Firmen der Halbleiterund Elektronikindustrie und bei den Herstellern von Laser- und Mikrowellengeräten, Computern und Hochpräzisionsinstrumenten (Saxenian 1983). Trotz des beeindruckenden Arbeitsplatzwachstums wurde die Regionalentwicklung nicht immer in der Weise beeinflußt, wie es die Strukturpolitik vorsah (dazu U.S. Congress. Congressional Budget Office 1985). 


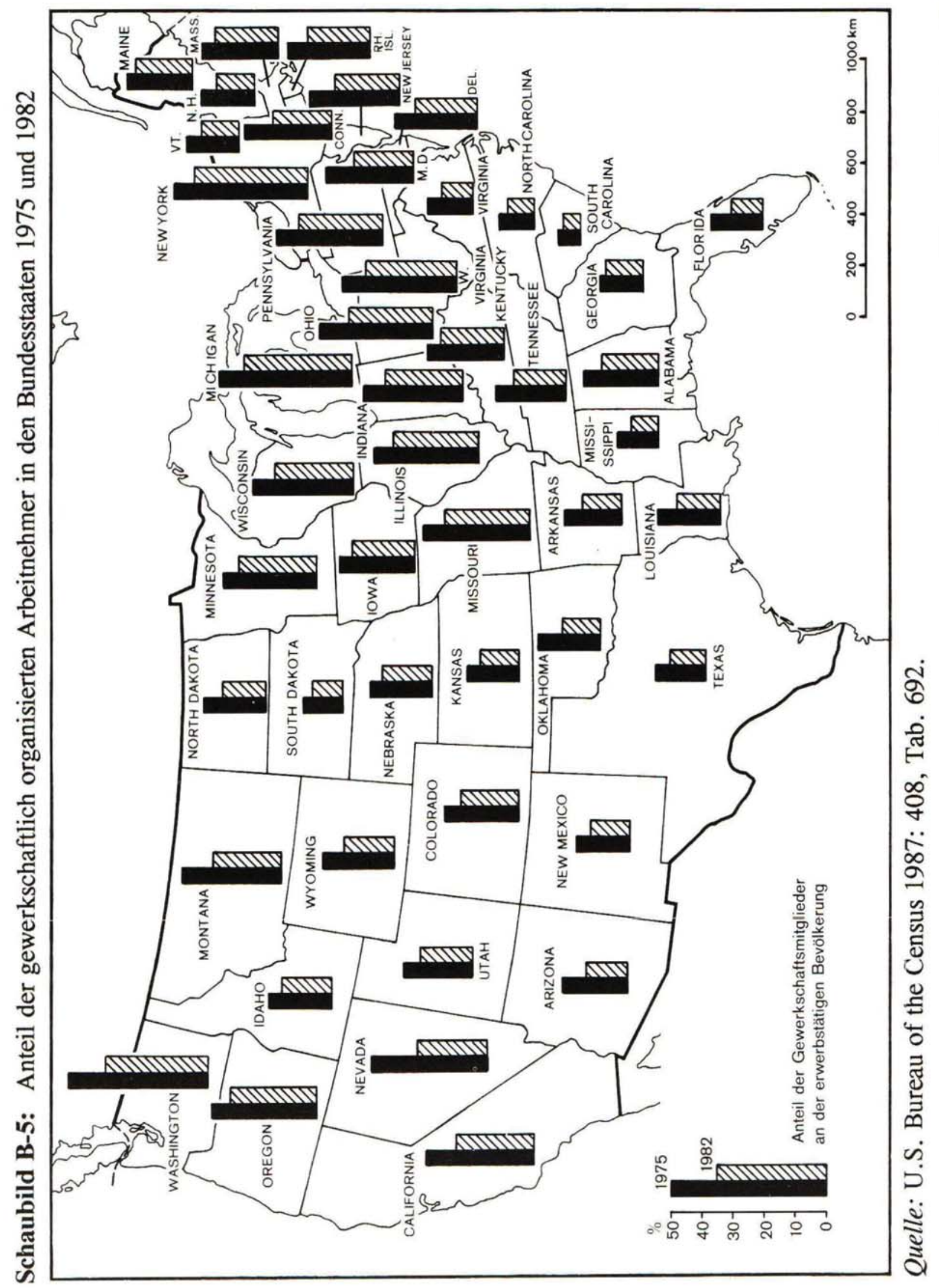




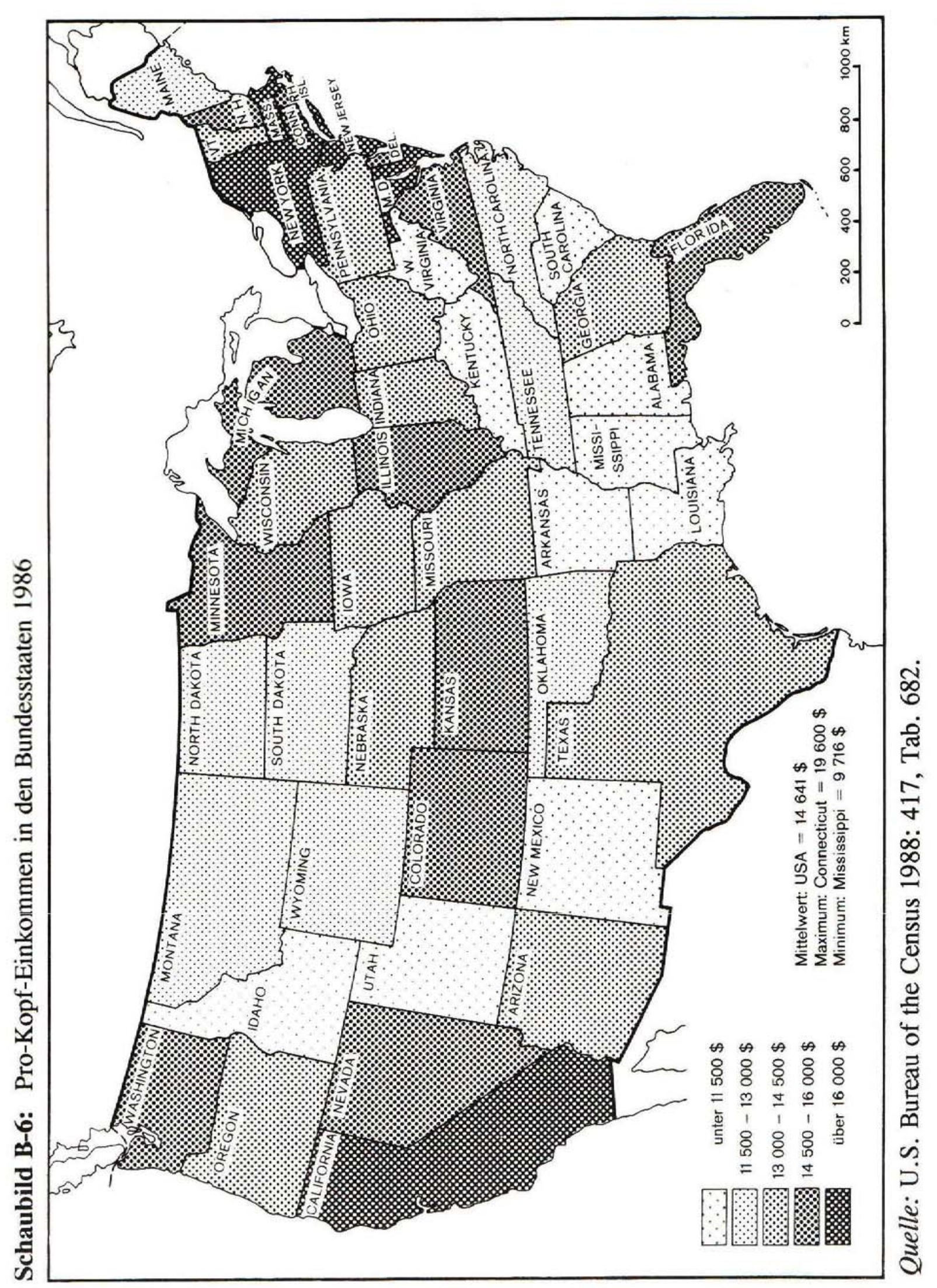


Obwohl Hochtechnologieindustrien weder hinsichtlich der Produktionsfaktoren noch des Marktes ortsgebunden sind, treten sie oft nur als spin-off von regierungseigenen Forschungszentren, Laboratorien und berühmten Universitäten in deren Umgebung auf, vor allem wegen des dort verfügbaren Pools von hochqualifizierten Technikern und Wissenschaftlern, Ausbildungsstätten und Faktoren der Lebensqualität (Premus 1982; Farrell 1982; Markusen/Hall/ Glasmeier 1986). Als Industrien mit dualem Arbeitskräftebedarf (in der Fertigung Billiglohnarbeiter, in der Forschung und Entwicklung wenige hochqualifizierte und hochbezahlte Fachkräfte) kann der direkte Einfluß der Hochtechnologiekolonien auf die Prosperität der Gesamtregion relativ gering sein. Ihr wichtigster multiplier effect

... may be in the form of jobs directly created through the demands of high technology employees ... failing some positive state intervention, a relatively few favored places will generate a modest number of high technology jobs and a much larger number of service jobs dependent on them (Hall/Markusen 1985: 144).

Als footloose industries (Premus 1982; Armington/Harris/Odle 1983) mit relativ hohem Mobilitätsgrad können sie außerdem je nach Gegebenheit ebenso leicht einen positiven, wie einem negativen multiplier effect herbeiführen, wie es gegenwärtig in einigen Städten des sunbelt der Fall ist (Sternlieb 1986: 211 220).

Abschließend lassen sich drei Tatsachen herausstellen:

(1) Die gegenwärtige industriegeographische Struktur zeigt einen relativ bedeutenden Nordosten, dessen traditionelle Industrien zwar hinsichtlich der Wertschöpfung und der Beschäftigtenzahl rückläufig sind, der aber in anderen Wirtschaftszweigen einen Aufschwung erlebt; der pazifische Nordwesten, der Westen und der Südwesten weisen verstärkt sog. Wachstumsindustrien auf, die Spitzenverdienste und eine regionale Einkommenssteigerung erlauben, deren Haupteffekt aber vor allem in der Schaffung von Arbeitsplätzen in den Billiglohnkategorien des Tertiärsektors liegen kann. Tatsächlich zeigen die 80er Jahre hier ein z.T. stark verlangsamtes Wachstum der Realeinkommen (Schneider-Sliwa 1989). Der Alte Süden ist in erster Linie durch arbeitsintensive Niedriglohnindustrien ausgewiesen, die das Bild vom sunbelt zu sun spots reduzieren können (Berentsen 1987: 467 und Schaubilder B-6 und B-7).

(2) Bemerkenswert bei der Ausbildung neuer industrieller Standorte ausserhalb des manufacturing belt ist, daß es sich dabei größtenteils nicht um das Abwandern von Industrien aus dem Nordosten in diese Gebiete handelt (Clark 1985: 104f; Rees 1978, 1979; Rees/Weinstein 1983). Untersuchungen von Standortentscheidungen und Firmenneugründungen ergeben, daß

... locally based firms and new firms, as opposed to external sources were responsible for most of the manufacturing 


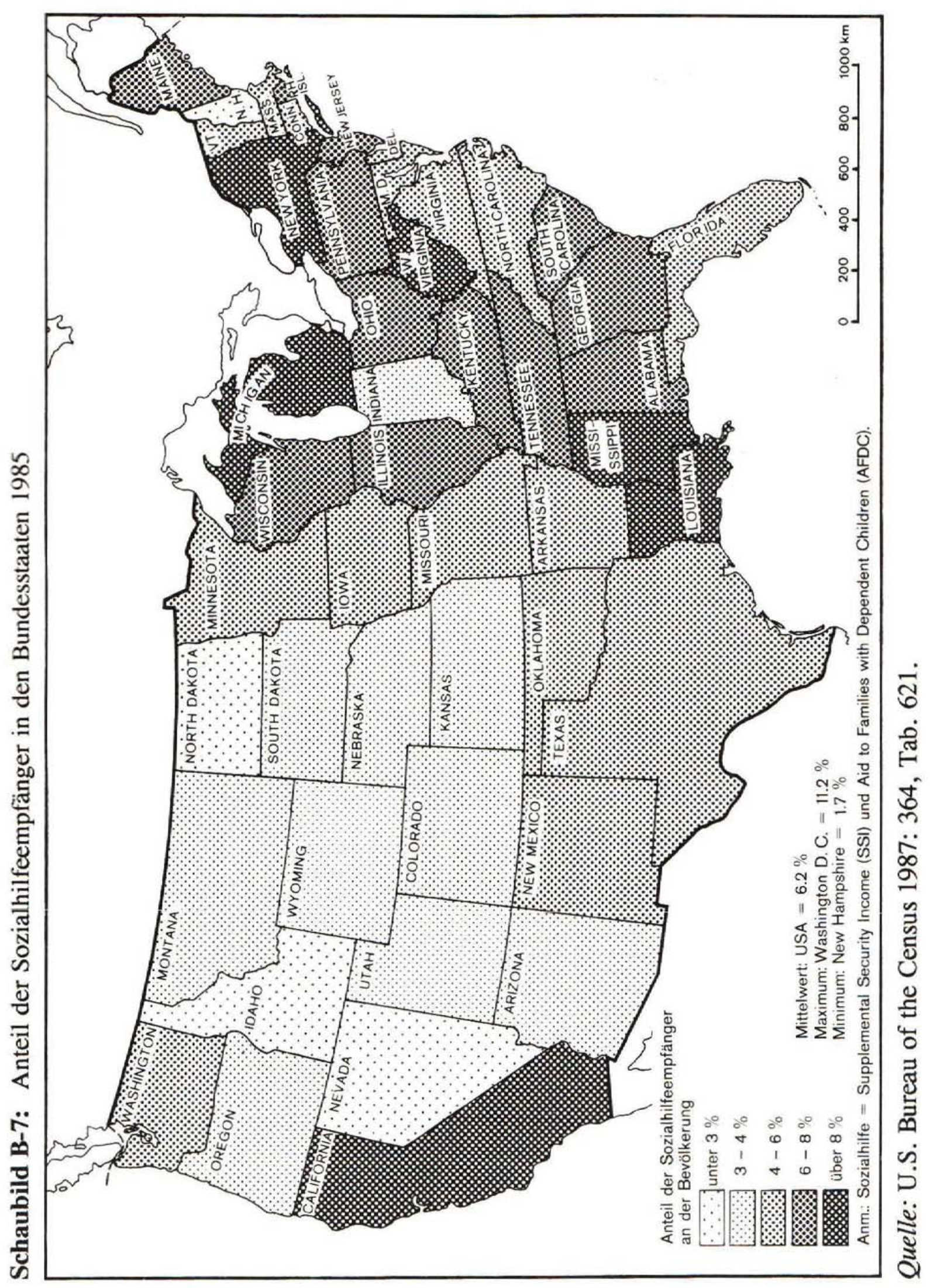


Strukturen der Wirtschaft

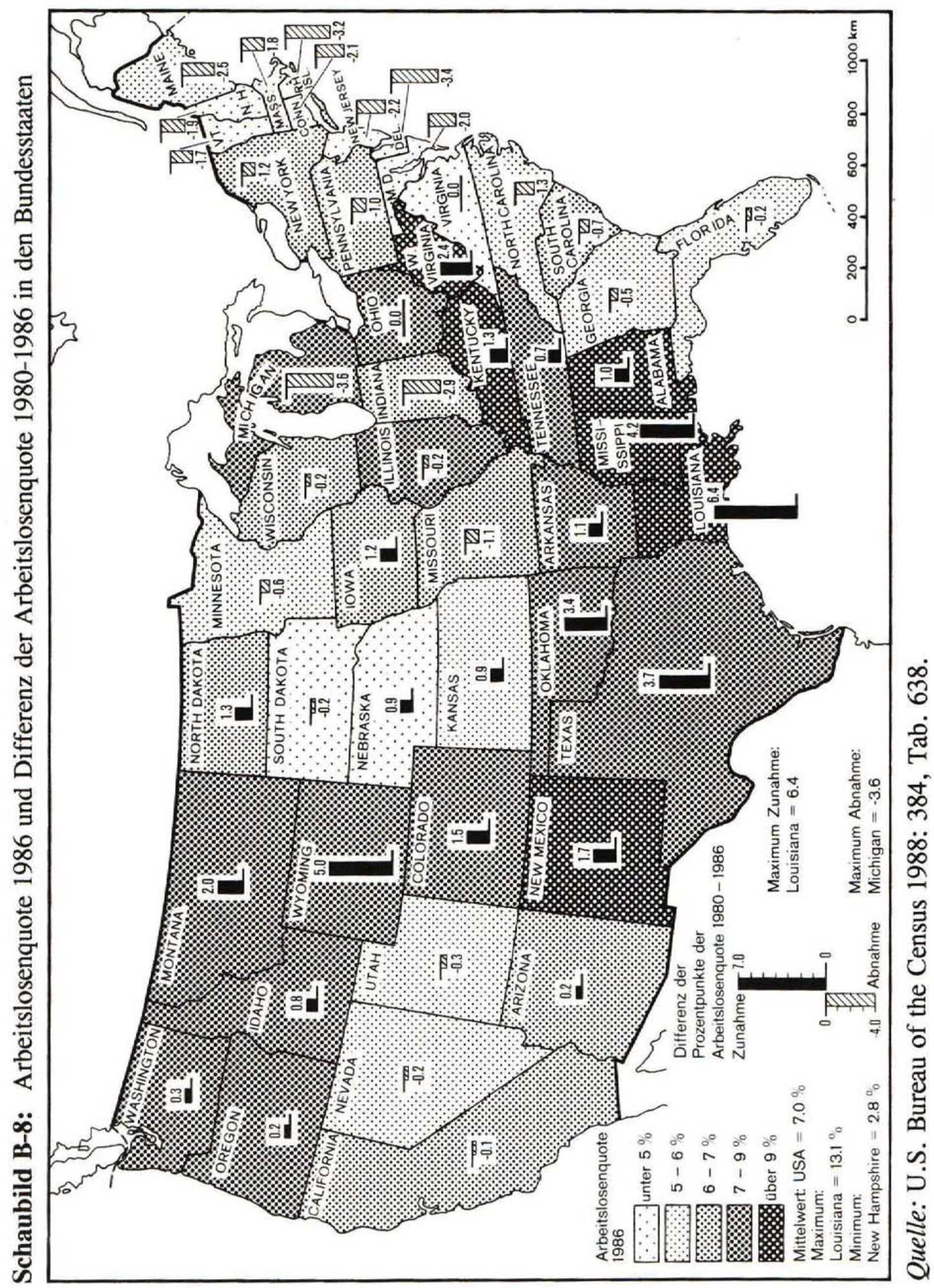


growth ... Decline in the Northeast does not automatically mean expansion in the South. The South is increasing in importance as a manufacturing region because it is both attractive to new firms and is an area which is conducive to industrial expansion and growth (Clark 1985: 104),

wobei Absatzmarktnähe, Verfügbarkeit der physischen Infrastruktur und des human capital, sowie ein unternehmerfreundliches Klima besonders wichtig sind (De Lange 1986: 112-125).

(3) Das gute regionale Image der neuen Industriestandorte ist in entscheidender Weise durch staatliche Investitionen und Interventionen begründet worden, die das Wirtschaftswachstum in der ehemals strukturschwachen "Peripherie", d.h. den Regionen außerhalb des manufacturing belt, gefördert haben. Allerdings spiegeln die tatsächlichen und regional spezifischen Arbeitsplatzveränderungen in hohem Maße noch bestehende oder neue wirtschaftliche und soziale Probleme wider und geben Denkanstöße für die Planung auf Bundes-, Einzelstaaten- und Kommunalebene.

\section{Der Tertiärsektor}

Der Eintritt in die "postindustrielle" oder Dienstleistungsgesellschaft wird vor allem durch das Ansteigen der Zahl von Arbeitsplätzen in der öffentlichen Verwaltung, dem Gesundheits- und Erziehungswesen, der Forschung und dem Hochtechnologiebereich (research and development, kurz R\&D genannt) seit den 50er Jahren angesetzt. Bell (1973) sieht den Umbruch in der Gesellschaft durch verschiedene Merkmale gekennzeichnet, z.B. ein Bedürfnis nach mehr Lebensqualität und staatlicher Fürsorge, eine allgemeine Zukunftsausrichtung der technischen Entwicklung und Betonung der Grundlagenforschung gegenüber einer ad-hoc-Anpassung an die Bedürfnisse der Konsumgüterindustrie, schließlich durch eine Konzentration der technologischen Entwicklung auf das Informationswesen vis-à-vis der früheren Ausrichtung auf Schwerindustrie, Maschinenbau und Energieträger. Ferner kennzeichnet die postindustrielle Gesellschaft eine Richtungsweisung durch Universitäten, Forschungsinstitute, Hochtechnologieentwicklungen, Wissenschaftler, Techniker und Akademiker im medizinischen und nichtmedizinischen Bereich, während im Industriezeitalter die Unternehmer des big business dominierten (Clark 1985: 7-10). Dieser gesellschaftliche Wandel hat sich auch auf die wirtschaftsgeographische Struktur ausgewirkt.

Der Tertiarisierungsgrad der Wirtschaft und seine regionale Verteilung gehen aus Tabelle B-2A hervor. Von den 97,614 Mio. nicht in der Landwirtschaft Beschäftigten waren 1985 74,5\% (75,684 Mio.) im tertiären Sektor und 19,8\% (19,312 Mio.) in der Industrie tätig. Wie bei der Industrialisierung ergibt sich beim Wachstum des Dienstleistungssektors eine gewisse Standortkonzentration. Diese ist z.T. aus der Marktabhängigkeit tertiärer Aktivitäten und der Bevölkerungskonzentration zu erklären und zeigt sich besonders in den 
Regionen Middle und South Atlantic, East North Central und Pacific bei den fünf Hauptkategorien des Tertiärsektors (vgl. Tabelle B-2B) sowie bei einer Aufgliederung der in Tabelle B-2 verwendeten Kategorie "übrige Dienstleistungen" (vgl. Tabelle B-3).

Tabelle B-2: Anteil der unselbständig Beschäftigten in Dienstleistungssektoren 1985

\begin{tabular}{|c|c|c|c|c|c|c|c|}
\hline & & & A. Sekt & rale Ante & & & \\
\hline & $\begin{array}{l}\text { Trans- } \\
\text { port u. } \\
\text { öfftl. } \\
\text { Versor- } \\
\text { gung }\end{array}$ & Handel & $\begin{array}{l}\text { Banken, } \\
\text { Immobi- } \\
\text { lien, Ver- } \\
\text { siche- } \\
\text { rung }\end{array}$ & $\begin{array}{l}\text { Übrige } \\
\text { Dienst- } \\
\text { leistun- } \\
\text { gen }\end{array}$ & $\begin{array}{l}\text { Öfftl. } \\
\text { Verwal- } \\
\text { tung }\end{array}$ & $\begin{array}{l}\text { Tertiär- } \\
\text { sektor } \\
\text { (gesamt) }\end{array}$ & $\begin{array}{l}\text { Nicht- } \\
\text { landwirt. } \\
\text { Beschäf- } \\
\text { tigte } \\
\text { (gesamt) }\end{array}$ \\
\hline $\begin{array}{l}\text { Mio. } \\
\%\end{array}$ & $\begin{array}{l}5,242 \\
5,4\end{array}$ & $\begin{array}{l}23,100 \\
23,7\end{array}$ & $\begin{array}{l}5,953 \\
6,1\end{array}$ & $\begin{array}{l}21,974 \\
22,5\end{array}$ & $\begin{array}{l}16,415 \\
16,8\end{array}$ & $\begin{array}{l}72,684 \\
74,5\end{array}$ & $\begin{array}{l}97,614^{a} \\
100,0\end{array}$ \\
\hline & & & B. Region & le Anteile & & & \\
\hline NEn ${ }^{b}$ & 4,8 & 6,0 & 6,7 & 6,7 & 4,9 & & \\
\hline MAt & 16,8 & 15,2 & 19,7 & 18,3 & 15,6 & & \\
\hline ENC & 15,7 & 17,1 & 15,6 & 16,5 & 15,8 & & \\
\hline WNC & 8,2 & 7,8 & 7,0 & 7,2 & 7,8 & & \\
\hline SAt & 16,9 & 17,2 & 15,6 & 16,5 & 18,1 & & \\
\hline ESC & 5,1 & 5,3 & 4,2 & 4,4 & 6,2 & & \\
\hline WSC & 11,6 & 11,1 & 10,6 & 9,2 & 11,1 & & \\
\hline Mnt & 5,7 & 5,4 & 5,1 & 5,6 & 6,0 & & \\
\hline $\mathrm{Pac} \mathrm{C}$ & 13,7 & 14,3 & 15,0 & 14,7 & 14,2 & & \\
\hline & & C. Ve & teilung inne & halb der I & gionen (\%) & & Mio. \\
\hline NEn & 4,2 & 23,0 & 6,5 & 24,4 & 13,3 & 71,4 & 6,069 \\
\hline MAt & 5,5 & 22,1 & 7,4 & 25,2 & 16,1 & 76,3 & 15,910 \\
\hline ENC & 4,9 & 23,6 & 5,5 & 21,6 & 15,4 & 71,0 & 16,804 \\
\hline WNC & 6,0 & 25,1 & 5,8 & 22,0 & 18,0 & 76,9 & 7,162 \\
\hline SAt & 5,3 & 23,7 & 5,5 & 21,7 & 18,7 & 74,9 & 16,783 \\
\hline ESC & 5,0 & 22,6 & 4,6 & 18,2 & 19,0 & 69,4 & 5,369 \\
\hline wSC & 5,9 & 25,0 & 6,1 & 19,8 & 17,7 & 74,5 & 10,259 \\
\hline Mnt & 5,8 & 24,2 & 5,9 & 23,9 & 19,4 & 79,2 & 5,110 \\
\hline $\mathrm{Pac}^{\mathrm{C}}$ & 5,2 & 24,1 & 6,5 & 23,5 & 17,1 & 76,4 & 14,356 \\
\hline
\end{tabular}

Quelle: Nach U.S. Bureau of the Census 1987: 395, Tab. 671.

a In Millionen, davon waren 1985 in der Industrie 19,312 (19,8\%) tätig.

b Regionen: New England (NEn), Middle Atlantic (MAt), East North Central (ENC), West North Central (WNC), South Atlantic (SAt), East South Central (ESC), West South Central (WSC), Mountain (Mnt), Pacific (Pac). Die Prozentangaben beziehen sich auf die erste Zahlenzeile des Teils A dieser Tabelle. Pacific ohne Alaska und Hawaii. 
Tabelle B-3: Regionale Anteile (\%) an den Beschäftigten in ausgewählten steuerpflichtigen Dienstleistungen: business services ${ }^{\mathrm{a}}$, personal services $^{\mathrm{b}}$, automotive repair $\mathrm{c}, 1982$

\begin{tabular}{lccc}
\hline & business & personal & $\begin{array}{c}\text { automotive } \\
\text { repair }\end{array}$ \\
\hline New England & 5,9 & 6,2 & 5,6 \\
Middle Atlantic & 17,7 & 16,4 & 15,8 \\
East North Central & 16,3 & 17,4 & 16,0 \\
West North Central & 6,0 & 8,3 & 7,6 \\
South Atlantic & 15,3 & 17,0 & 15,9 \\
East South Central & 3,6 & 5,9 & 5,3 \\
West South Central & 11,0 & 10,0 & 11,3 \\
Mountain & 5,6 & 5,1 & 5,8 \\
Pacific (ohne Alaska & 17,3 & 13,1 & 16,0 \\
$\quad$ und Hawaii) & & & \\
\hline
\end{tabular}

Quelle: Nach U.S. Bureau of the Census 1989: 768, Tab. 1353 (veränd.).

a Dazu gehören die im Standard Industrial Classification Code (SIC) mit der Nummer 73 gekennzeichnete Branchen wie z.B. Werbung und Werbeagenturen, EDV- und Computer-Consulting-Firmen, Programmierungsund Software-Entwicklungsbetriebe sowie alle Computerfirmen außer den Produktionsbetrieben, Management, Consulting- und Public-RelationsFirmen, Arbeitsvermittlungsbüros, Detekteien, Wach- und Schließgesellschaften.

b SIC Codenummer 72: Wäschereien, Schneidereien, Fotostudios, Friseursalons, Bestattungsunternehmen u.a.

c SIC Codenummer 75: Autoreperaturwerkstätten, Fahrzeugverleih u.ä.

Nicht alle Standortkonzentrationen tertiärer Aktivitäten sind bevölkerungs- und marktbedingt, sondern z.T. auch das Resultat staatlicher Planung für periphere Räume, die weit entfernt von den großen Bevölkerungs- und Marktzentren liegen. Untersucht man z.B. die Aufgliederung des Tertiärsektors innerhalb der einzelnen geographischen Regionen (vgl. Tabelle B-2C) und der USA insgesamt (vgl. Tabelle B-2A), so fallen in einigen Regionen eine Überbzw. Unterrepräsentierung einzelner Dienstleistungsgruppen auf, gemessen am entsprechenden nationalen Wert. Eine Unterrepräsentierung aller Dienstleistungsbereiche mit Ausnahme der öffentlichen Verwaltung fällt für den Bereich East South Central auf (Kentucky, Tennessee, Mississippi, Alabama). Dies ist eines der wirtschaftlich rückständigen Gebiete, das man verstärkt seit 1961 durch den Area Redevelopment Act und sein Nachfolgegesetz, den Economic Development Act von 1965, zu fördern suchte. Die Überrepräsentierung der öffentlichen Verwaltung hier wie auch in South Atlantic ist in diesem Zusammen- 
hang zu sehen und auf den geplanten Ausbau von bundeseigenen "Firmen" (NASA, Militäreinrichtungen, Forschungszentren, Auslagerungen von Bundesbehörden) zurückzuführen (Clark, 1985: 80). Obwohl eine solche Konzentration der öffentlichen Verwaltung als Entwicklungsindikator angesehen wird und oft als sog. growth pole geschaffen wurde, ist ihre eigentliche Auswirkung auf die Regionalentwicklung oft sehr begrenzt. Vor allem dort, wo öffentliche Verwaltung zum großen Teil militärische Anlagen, Stützpunkte und Beschäftigte umfaßt (North Carolina, Georgia, Alabama, Virginia), werden wenig trickle-down effects spürbar. Die Tausende von Beschäftigten, die sich im Verlauf eines Jahres auf solchen Anlagen aufhalten, können ihre Nachfrage nach Unterkünften und täglicher Lebenshaltung durch ein bundeseigenes, subventioniertes und unter dem Marktpreis liegendes Angebot decken (Birdsall/Florin 1981: 246). Auch die nicht unerheblichen Investitionen der Bundesregierung (vgl. Tabelle B-4), die allerdings von großer nationaler Bedeutung sind, wirken sich oft mehr auf einzelne Städte aus als auf den Bundesstaat oder die Gesamtregion, so daß auch in den 80er Jahren weite Teile von East South Central und South Atlantic als ausgesprochen arme Gebiete gelten (vgl. Schaubild B-6 und B-7).

Betrachtet man die Überrepräsentierung der öffentlichen Verwaltung in der dünn besiedelten Mountain-Region (vgl. Tabelle B-2C), so ist diese durch den hohen Anteil der Beschäftigten in bundeseigenen Behörden und R\&D-Stätten zu erklären, ferner durch die Arbeitsplätze, die mit der Erhaltung der grossen Naturparks in Idaho, Wyoming, Utah oder Arizona verbunden sind (Clark 1985: 76). Verhältnismäßig wenig öffentliche Verwaltung fällt in den altindustrialisierten Gebieten New England und East North Central auf (vgl. Tabelle B-2C), die als Teil des ehemaligen wirtschaftlichen Kernlandes der USA nicht im selben Maße wie andere Regionen, z.B. der Alte Süden, der regionalplanerischen Intervention der Bundesregierung mit gleichzeitigem Wachstum des federal employment bedurfte. Dies wird auch bei der Betrachtung der anderen Dienstleistungskategorien ersichtlich. So ist z.B. New Englands Anteil an "übrigen Dienstleistungen", zu denen auch Hochtechnologiedienstleistungen zählen, höher als der entsprechende nationale Wert $(24,4 \%$ gegenüber $22,5 \%$, vgl. Tabellen B-2C und B-2A), und weist diese als wirtschaftlichen Grundpfeiler der Region aus. Demgegenüber zeigen East South Central und z.T. West South Central durch schwächere Ausbildung fast aller tertiärer Bereiche außer der öffentlichen Verwaltung eine allgemeine "Untertertiarisierung" im Vergleich zum entsprechenden nationalen Durchschnitt und damit ihre Abhängigkeit von der öffentlichen Hand als Arbeitgeber. Obwohl hier die öffentliche Hand einen großen Anteil an der Beschäftigtenzahl hat, darf man längerfristig gesehen die Rolle des Bundes und der Einzelstaaten nicht überbewerten. Bınd und Staaten werden oft nur indirekt oder mit geringen Resultaten wirksam (mit Ausnahme der Tennessee Valley Authority). Von Bund und Ländern betriebene Strukturpolitik mit Subventionen oder steuerlichen Anreizen (dazu McGuire 1986) ist vielfach nur Vorbereitung zur Attraktivitätssteigerung von Standorten, wobei da- 
nach das Handeln der Privatwirtschaft überlassen bleibt. Diese übernimmt das meist nicht in dem Maße wie es erwartet wird oder notwendig ist, um Regionen aus Stagnation, Unterentwicklung und Armut herauszuführen (Vollmar 1986).

Tabelle B-4: Regionale Verteilung und Nettowert von Aufträgen des Verteidigungsministeriums für Forschung, technologische Entwicklung, Tests und Auswertung an die 50 größten Vertragspartner, Fiskaljahr 1985 (Mio. \$) $^{\mathrm{a}}$

\begin{tabular}{|c|c|c|c|c|c|}
\hline \multicolumn{2}{|c|}{ New England } & \multicolumn{2}{|c|}{ Middle Atlantic } & \multicolumn{2}{|c|}{ East North Central } \\
\hline $\begin{array}{l}\text { NH } \\
\text { VT } \\
\text { M } \\
\text { RI } \\
\text { CT }\end{array}$ & $\begin{array}{r}1,2 \\
5,8 \\
987,1 \\
21,1 \\
170,8\end{array}$ & $\begin{array}{l}\text { NY } \\
\text { NJ } \\
\text { PA }\end{array}$ & $\begin{array}{r}1910,5 \\
509,5 \\
235,9\end{array}$ & $\begin{array}{l}\mathrm{OH} \\
\text { IN } \\
\text { MI }\end{array}$ & $\begin{array}{r}182,1 \\
10,6 \\
138,3\end{array}$ \\
\hline \multicolumn{2}{|c|}{ West North Central } & \multicolumn{2}{|c|}{ South Atlantic } & \multicolumn{2}{|c|}{ East South Central } \\
\hline $\begin{array}{l}\text { MI } \\
\text { IA } \\
\text { MC } \\
\text { KS }\end{array}$ & $\begin{array}{r}249,7 \\
47,7 \\
430,7 \\
98,9\end{array}$ & $\begin{array}{l}\text { MD } \\
\text { DC } \\
\text { VA } \\
\text { WV } \\
\text { NC } \\
\text { GA } \\
\text { FL }\end{array}$ & $\begin{array}{r}405,3 \\
1,7 \\
401,8 \\
0,7 \\
62,2 \\
15,6 \\
425,9\end{array}$ & $\begin{array}{l}\text { KY } \\
\text { TN } \\
\text { AL }\end{array}$ & $\begin{array}{r}0,3 \\
44,1 \\
107,2\end{array}$ \\
\hline \multicolumn{2}{|c|}{ West South Central } & \multicolumn{2}{|c|}{ Mountain } & Pacif & \\
\hline $\begin{array}{l}\text { AR } \\
\text { OK } \\
\text { TX }\end{array}$ & $\begin{array}{c}0,7 \\
0,06 \\
725,1\end{array}$ & $\begin{array}{l}\text { CO } \\
\text { NM } \\
\text { AZ } \\
\text { UT } \\
\text { NV }\end{array}$ & $\begin{array}{r}615,0 \\
65,2 \\
118,5 \\
203,6 \\
1,6\end{array}$ & $\begin{array}{l}\text { WA } \\
\text { CA } \\
\text { HI }\end{array}$ & $\begin{array}{r}952,3 \\
4439,6 \\
6,4\end{array}$ \\
\hline
\end{tabular}

Quelle: Nach U.S. Department of Defense 1985: Tab. 2.

a Werte sind auf- bzw. abgerundet.

$\mathrm{Da} ß$ die relative Unterentwicklung des tertiären Sektors im Alten Süden mit der allgemeinen politischen, soziologischen und historischen Entwicklung zusammenhängt und weriger von der noch vorherrschenden agraren Basis, wird bei einem Vergleich mit West North Central deutlich; diese Region gehört zum agraren Kernland der USA und ist trotzdem stark tertiärwirtschaftlich ausgerichtet. Im Handel lag der Anteil 1985 mit 25,1\% sogar wesentlich höher als der nationale Wert von 23,7\%, was z.T. auf die enge Beziehung von Landwirtschaft und Vermarktung zurückzuführen ist.

Untertertiarisiert sind im allgemeinen auch noch die altindustrialisierten Gebiete wie New England (trotz des erwähnten Hochtechnologieschwerpunktes, der Dienstleistungen mit einschließt), sowie East North Central mit 71,4\% bzw. $71 \%$. Allerdings liegt im ebenfalls altindustrialisierten Middle Atlantic der Dienstleistungssektor mit $76,3 \%$ der Beschäftigten deutlich über dem nationalen 
Wert. Für die altindustrialisierten Regionen darf man in Zukunft einen noch stärkeren und von den Staaten finanziell geförderten Ausbau der Dienstleistungsbereiche zum Ausgleich für rückläufige Industrien erwarten. Bestehende Maßnahmen in dieser Richtung sind durch Tabelle B-5 angezeigt, wonach z.B. in Middle Atlantic und East North Central einem größeren Anteil der Dienstleistungsbetriebe Steuerfreiheit gewährt wird (wenn auch nur anfänglich) als im übrigen Staatsgebiet. Dies folgt zum Teil dem erfolgreichen Ansatz der Neuenglandstaaten, die Ende der 70er bis Anfang der 80er Jahre Wirtschaftsförderung durch Steuervergünstigungen auf der Grundlage jobs first, equity later betrieben (Sternlieb 1986). Dabei spielen auch Erkenntnisse über den möglichen Zusammenhang zwischen Steuerpolitik und Migrationsentscheidungen eine Rolle. Planungsmaßnahmen in South Atlantic versuchen ebenfalls verstärkt und z.T. über Steuervergünstigungen, im Umkreis von bundeseigenen Firmen Hochtechnologiedienstleistungsbetriebe anzusiedeln.

Tabelle B-5: Anteil (\%) der steuerfreien Dienstleistungsbetriebe in geographischen Regionen 1982

\begin{tabular}{lr}
\hline New England & 7,6 \\
Middle Atlantic & 16,8 \\
East North Central & 17,7 \\
West North Central & 8,5 \\
South Atlantic & 15,0 \\
East South Central & 5,0 \\
West South Central & 8,2 \\
Mountain & 5,4 \\
Pacific (ohne Alaska & 13,6 \\
und Hawai) & \\
\hline
\end{tabular}

Quelle: Nach U.S. Bureau of the Census 1989: 768, Tab. 1353.

Wie stark die Planung auf die Tertiarisierung eines Raumes einwirken kann, wird am Beispiel Pittsburghs deutlich, das aufgrund jahrelanger Maßnahmen 1985 erstmalig mehr Menschen in seinen neuen high-tech- und Dienstleistungsbetrieben beschäftigte als in der Stahlindustrie. Im Hinblick auf den Rückgang der Stahlindustrie von Pittsburgh, der sich mit der endgültigen Zerschlagung des Preismonopols seit 1948 abzeichnete und danach durch den allgemeinen postindustriellen Strukturwandel beschleunigt wurde und zwischen den fünfziger und achtziger Jahren zum Verlust von 110.000 Arbeitsplätzen in der Stahlindustrie führte, hatte die Stadt schon seit den vierziger Jahren durch eine Planungskoalition der Privatindustrie mit der öffentlichen Hand (Allegheny Conference on Community Development) eine Umstrukturierung versucht. Mittels eines neuen Stadtentwicklungsplans wurde eine Imageaufwertung betrieben, bei der in der Innenstadt moderne Bürohochhäuser Rangierbahnhöfe und 
Fabrikhallen ablösten (Lubove 1969). In der Folge siedelten sich viele Konzernverwaltungen an, die das Steueraufkommen und die Wirtschaft der Stadt entsprechend stärkten. Ferner kamen rund 300 Biotechnologie-, Software- und andere Hochtechnologiefirmen hinzu, die teils durch die bedeutenden Universitäten der Stadt angeworben und teils durch das Pentagon unterstützt wurden (Deysson 1988: 44f.; Economou 1987: 105-146).

Tabelle B-6: Anzahl der Konzernverwaltungen in ausgewählten Städten 1m Süden 1960-1980

\begin{tabular}{lrrr}
\hline & 1980 & 1970 & 1960 \\
\hline Houston & 23 & 7 & 6 \\
Dallas-Ft. Worth & 23 & 14 & 15 \\
Atlanta & 7 & 6 & 3 \\
Greensboro- & & & \\
Winston-Salem & 8 & 6 & 5 \\
Richmond & 7 & 4 & 7 \\
Miami & 5 & 2 & 2 \\
Charlotte & 3 & 2 & 1 \\
New Orleans & 3 & 1 & 2 \\
Oklahoma City & 1 & 1 & 3 \\
Tampa & 2 & 1 & 0 \\
Birmingham & 2 & 2 & 3 \\
\hline
\end{tabular}

Quelle: Nach Wheeler und Brown 1985: 71.

Ähnliche Planungen werden sicherlich mit Erfolg in anderen altindustrialisierten Städten des manufacturing belt als Anpassung an den allgemeinen Strukturwandel durchgesetzt werden. Dabei haben diese Städte gute Voraussetzungen. Erstens haben rund zwei Drittel der $\mathbf{5 0 0}$ größten Konzerne weiterhin ihre Hauptverwaltungen in diesen Städten, obwohl auch die Städte des sunbelt Konzernverwaltungen anzuziehen versuchen (vgl. Tabelle B-6).

$Z$ weitens vereinen altindustrialisierte Regionen schon eine große Anzahl von high-tech-Dienstleistungsbetrieben auf sich (vgl. Tabelle B-7). Dies ist dadurch bedingt, daß fast alle Industrieprodukte des manufacturing belt stark R\&D-intensiv sind (Davis 1982). Daher unterhalten viele Industrien ihre eigenen Forschungslabors in Großstädten mit spezialisiertem Arbeitsmarkt und Betrieben, in denen die technologische Entwicklung direkt in die Produktion umgesetzt werden kann, oder in der Nähe von Universitäten, deren Forschungsprogramme zum Teil auf Innovationen für ansässige Industrien ausgerichtet sind (Clark 1985: 97). Eine Verdichtung von R\&D-Laboren und -Angestellten besteht daher für alle atlantischen Metropolen von Boston bis Washington; außerhalb des östlichen Randes des manufacturing belt gibt es nur vereinzelt 
Tabelle B-7: Führende Bundesstaaten in ausgewählten Hochtechnologiebereichena,b 1985

\begin{tabular}{|c|c|c|c|c|c|c|c|}
\hline \multirow[t]{3}{*}{ A. Computer } & & \multicolumn{4}{|l|}{ B. Halbleiter } \\
\hline & \multicolumn{2}{|c|}{ Anzahl der } & \multirow{2}{*}{$\begin{array}{l}\% \\
\text { US }\end{array}$} & & \multicolumn{2}{|c|}{ Anzahl der } & \multirow{2}{*}{$\begin{array}{l}\% \\
\text { US }\end{array}$} \\
\hline & Firmen & $\begin{array}{l}\text { Beschäf- } \\
\text { tigten }\end{array}$ & & & Firmen & $\begin{array}{l}\text { Beschäf- } \\
\text { tigten }\end{array}$ & \\
\hline Kalifornien & 613 & 157063 & 34,8 & Kalifornien & 365 & 72303 & 35,0 \\
\hline Massachusetts & 153 & 50924 & 11,3 & Texas & 56 & 39540 & 19,2 \\
\hline New York & 96 & 32303 & 7,2 & Arizona & 29 & 17348 & 8,4 \\
\hline Texas & 80 & 26692 & 6,6 & Florida & 33 & 9843 & 4,8 \\
\hline Minnesota & 52 & 27831 & 6,2 & Pennsylvania & 33 & 9589 & 4,6 \\
\hline Arizona & 24 & 19618 & 4,3 & Massachusetts & 72 & 9134 & 4,4 \\
\hline Colorado & 34 & 16522 & 3,7 & Colorado & 28 & 6270 & 3,0 \\
\hline North Carolina & 26 & 16299 & 3,6 & Oklahoma & 4 & 6100 & 3,0 \\
\hline \multirow{2}{*}{ Pennsylvania } & 51 & 9850 & 2,2 & Ohio & 17 & 3629 & 2,7 \\
\hline & & & & New York & 95 & 5337 & 2,6 \\
\hline \multicolumn{4}{|c|}{$\begin{array}{l}\text { C. Chirurgische und medizinische } \\
\text { Instrumente }\end{array}$} & \multicolumn{4}{|c|}{ D. Programmierung und Software } \\
\hline & \multicolumn{2}{|c|}{ Anzahl der } & $\%$ & & \multicolumn{2}{|c|}{ Anzahl der } & $\%$ \\
\hline & Firmen & $\begin{array}{l}\text { Beschäf- } \\
\text { tigten }\end{array}$ & US & & Firmen & $\begin{array}{l}\text { Beschäf- } \\
\text { tigten }\end{array}$ & US \\
\hline Kalifornien & 140 & 15215 & 18,8 & Kalifornien & 578 & 22937 & 20,2 \\
\hline New Jersey & 51 & 7789 & 9,6 & Texas & 237 & 10685 & 9,4 \\
\hline Ohio & 14 & 6093 & 7,5 & Virginia & 128 & 9304 & 8,2 \\
\hline Massachusetts & 48 & 5796 & 7,2 & Massachusetts & 166 & 8712 & 7,7 \\
\hline Illinois & 41 & 5666 & 7,0 & New York & 227 & 8231 & 7,2 \\
\hline New York & 46 & 5055 & 6,2 & Florida & 97 & 5982 & 5,3 \\
\hline Pennsylvania & 41 & 4283 & 5,3 & Maryland & 101 & 5684 & 5,0 \\
\hline Utah & 6 & 3965 & 4,7 & New Jersey & 132 & 4578 & 4,0 \\
\hline Connecticut & 29 & 3828 & 4,7 & Illinois & 147 & 4197 & 3,7 \\
\hline Colorado & 19 & 3331 & 4,1 & Pennsylvania & 100 & 3467 & 3,1 \\
\hline Florida & 19 & 2757 & 3,4 & Georgia & 79 & 3402 & 3,0 \\
\hline Missouri & 18 & 2459 & 3,0 & Ohio & 84 & 3385 & 3,0 \\
\hline Texas & 26 & 2449 & 3,0 & Michigan & 68 & 3306 & 2,9 \\
\hline Washington & 10 & 2221 & 2,7 & Colorado & 74 & 2691 & 2,4 \\
\hline Indiana & 16 & 1857 & 2,3 & & & & \\
\hline
\end{tabular}

Quelle: Nach Malecki 1985: 364f.

a Die vier Hochtechnologiebereiche fallen sowohl unter die Definition einer Industrie als auch unter die Kategorie der Dienstleistungen aufgrund ihrer R\&D-Intensität, Prozentsatz der Wissenschaftler und Ingenieure und Anteil der Einnahmen, die in R\&D investiert sind. Für eine Diskussion über die Definition von Hochtechnologiebetrieben vgl. Malecki (1985) und Glasmeier (1987).

b Da nur die Staaten mit über $25 \%$ der Beschäftigten in diesem Sektor eingeschlossen sind, liegt die Summe der Prozente unter $100 \%$. 
derartige Zentren, wie in Lafayette (Indiana), Madison (Wisconsin), Denver/ Boulder (Colorado), San Francisco/San Jose und Los Angeles/San Diego (Kalifornien) und dem research triangle Raleigh/Durham/Chapel Hill (North Carolina) (Malecki 1980, 1981, 1985). Hinsichtlich der Computerproduktion und -entwicklung weisen altindustrialisierte Staaten (z.B. Massachusetts, New York, Pennsylvania) zusammen 20,7\% der Gesamtbeschäftigung auf, bei der Erstellung von Software insgesamt 44,8\% (Virginia, Massachusetts, New York, Maryland, New Jersey, Illinois, Pennsylvania, Ohio und Michigan) und bei der Entwicklung von chirurgischen und medizinischen Instrumenten 44,8\% (vgl. Tabelle B-7).

Hinsichtlich dieser vier Hochtechnologiebranchen zählt mindestens eine altindustrialisierte Region neben Kalifornien und Colorado zu einer incubator area, von der wichtige Innovationen und Impulse für die Weiterentwicklung und Produktion ausgehen (Malecki 1985: 361-365). Insgesamt aber verlor der Nordosten zwischen 1973 und 1983 in diesen Bereichen Arbeitsplätze, wobei sich der Zuwachs auf den Westen konzentrierte. In der Computer- und Halbleiterentwicklung und -herstellung vereinte Kalifornien 1983 allein $34,8 \%$ bzw. $35 \%$ der gesamten Beschäftigung, bei medizinischen Instrumenten $18,8 \%$ und in der Softwareentwicklung 20,2\% (vgl. Tab B-7). Man kann daher zwar einen relativ starken Hochtechnologiedienstleistungssektor in altindustrialisierten Gebieten beobachten, aber auch eine regionale Verschiebung dieser Branchen in den sunbelt, vor allem nach Kalifornien. Dies muß jedoch nicht auf einen echten Bedeutungsverlust des Nordostens hinweisen, da die high-tech-Betriebe im sunbelt zu einem größeren Teil Filialen oder Auslagerungen von Firmen (branch plants) sind, die im Nordosten ihre Stammsitze haben (Koch u.a. 1983; Malecki 1985). Dies ist z.B. bei Computerfirmen der Fall, während die Firmen, die medizinische Instrumente entwickeln und herstellen, im altindustrialisierten Nordosten verbleiben (Malecki 1985: 358-361).

Beachtenswert ist, daß die R\&D-Stätten, die überwiegend durch die Privatwirtschaft finanziert und initiiert sind (ca. 50\% der gesamten R\&D-Ausgaben in den USA), sich vorwiegend im Nordosten und Westen der USA konzentrieren, während R\&D, das in bundeseigenen Zentren oder durch Vertragsvergabe der öffentlichen Hand getätigt wird, generell breiter gestreut ist, vor allem stadtspezifisch in den peripheren Räumen, die man im Rahmen der Regionalplanung nach einem gesamtstaatlichen Dispersionsverfahren fördern wollte. Neben Washington, D.C. mit einer großen Anzahl von R\&D-Beschäftigten in seinen Bundesbehörden und bundeseigenen Forschungszentren gibt es weitere Konzentrationen von regierungseigener R\&D im "space triangle" Houston (Texas)/Huntsville (Alabama)/Titusville-Cocoa Beach (Florida), ferner New Orleans (Louisiana), Oak Ridge-Knoxville (Tennessee) und Albuquerque (New Mexico) auf dem Energiesektor, in Pennsacola (Florida), Newport News/Hampton (Virginia) im Verteidigungssektor, in Dayton (Ohio) in der Luft- und Raumfahrt (Clark 1985: 95). Da Regierungsverträge aber auch "außer 
Haus" an Universitäten und die Industrie vergeben werden, sind bundesfinanzierte R\&D-Aktivitäten und -Angestellte ebenfalls in den großen Städten des Nordostens, des Mittelwestens und der Westküste vertreten, vor allem im Umfeld der wichtigsten Universitäten wie dem Massachusetts Institute of Technology, Harvard, Columbia, Johns Hopkins, Cornell, den Universitäten von Chicago, Wisconsin, Minnesota, Michigan, dem Staat Washington und in Kalifornien Berkeley, Stanford und UCLA (Clark 1985: 95-97).

Zusammenfassend läßt sich sagen, daß die Standorte der privatwirtschaftlichen R\&D-Aktivitäten in erster Linie von Marktprinzipien bestimmt sind, d.h. von Faktoren, die die Profitabilität beeinflussen, z.B. bestehenden Konzentrationen von Wissenschaftlem und Ingenieuren, Universitäten und Konzernverwaltungen (Malecki 1981; Massey 1984; Glasmeier et al. 1984: 166). Wo diese Faktoren in altindustrialisierten Regionen gegeben sind, finden sich Hochtechnologiekonzentrationen ebenso wie in den neuindustrialisierten Regionen des sunbelt. Das Verteilungsmuster der bundeseigenen und -finanzierten R\&D-Standorte zeigt neben den Marktprinzipien auch regionalplanerische Prinzipien, bei denen bevorzugt strukturschwache Räume gefördert wurden, sowie strategische bzw. verteidigungspolitische Aspekte, wonach sowohl Standorte in entlegenen Teilen des Landesinnern als auch an der Küste ausgebaut wurden.

\section{Der Agrarsektor}

Mit der Tertiarisierung der Wirtschaft verringerte sich auch die Bedeutung des Agrarsektors an der Gesamtbeschäftigung. Während 1960 noch 3,963 Mio. Farmen im Census erfaßt wurden, waren es 1974 nur noch 2,795 Mio. Nach der 1974 erfolgten Neudefinition von Farmbetrieben (alle Einheiten mit einer Agrarproduktion von mindestens $\$ 1.000$ jährlich) gab es 1975 2,521 Mio. Betriebe, 1985 2,275 Mio. und 1988 2,159 Mio. Farmen. Die Farmbevölkerung verringerte sich von 15,635 Mio. (1960, nach der 1969er Definition) auf 6,241 Mio. (1979, nach der 1974er Definition) und bis 1988 auf 4,951 Mio. Die Zahl der in der Landwirtschaft Beschäftigten sank von 3,774 Mio. (1979) auf 2,954 Mio. (1988). Wie Tabelle B-8 zeigt, erfolgte die Abnahme der Farmen nicht gleichmäßig, so waren die Regionen Mountain und Pacific im Zeitraum von 1980 bis 1988 nicht betroffen, während die traditionellen Farmgebiete East und West North Central sowie Middle und South Atlantic, East und West South Central die stärksten Einbußen erfuhren (Windhorst 1987; U.S. Bureau of the Census 1987: 620, 1989: 628, 1990: 637).

$\mathrm{Zu}$ den Faktoren, die in den einzelnen Regionen den Rückgang beschleunigten, gehören in East South Central und South Atlantic die schon angesprochene Neuindustrialisierung durch Niedriglohnbranchen, in West South Central der hohe Mechanisierungsgrad der Landwirtschaft, verbunden mit besser bezahlten Arbeitsplätzen in den übrigen Branchen. In den nördlichen Agrar- 
regionen wiegen neben der allgemeinen Mechanisierung der Landwirtschaft die damit verbundene Überschuldung der Farmen, schlechte Absatzmöglichkeiten oder Weltmarktpreise und Wertverlust des landwirtschaftlichen Grundbesitzes und niedrige Farmeinkommen besonders schwer (Windhorst 1987). Ferner trug in fast allen Regionen direkte oder indirekte Farmpolitik seit den 30er Jahren mit dazu bei, daß Farmen aufgegeben wurden, z.B. durch staatliche Bodenerhaltungsmaßnahmen in erosionsgefährdeten Gebieten. In jüngerer Zeit wurden vor allem marginalwirtschaftende Farmen, d.h. solche, die ohne Gewinn produzierten und durch staatliche Unterstützungsprogramme subventioniert worden waren, nach Absetzen dieser Programme verstärkt aufgegeben (Lutrell 1984; Clark 1985; Blume 1987, Bd. 1). Dieses "Farmsterben"

... masks the continuing, indeed increasing importance of agriculture in absolute terms. An expanding domestic population with sophisticated and varied eating habits imposes heavy demands upon the food producing sector. Moreover, agricultural products are of strategic importance in a world plagued by shortages and starvation. Although much diminished in relative terms, the agricultural sector forms a cornerstone of the post-industrial economy (Clark 1985: 143).

Tabelle B-8: Anzahl, Verteilung und prozentuale Abnahme der Farmen 1980-1988

\begin{tabular}{lrrrrr}
\hline & \multicolumn{2}{c}{1980} & \multicolumn{2}{c}{1988} & \\
& Zahl & $\%$ & Zahl & $\%$ & Abnahme \\
& $(2,43$ Mio. $)$ & & $(2,159$ Mio. $)$ & $(\%)$ \\
\hline New England & 30000 & 1,2 & 29000 & 1,3 & $-3,3 \%$ \\
Middle Atlantic & 118000 & 4,9 & 103000 & 4,8 & $-12,7$ \\
East North Central & 447000 & 18,4 & 379000 & 17,6 & $-15,2$ \\
West North Central & 562000 & 23,1 & 506000 & 23,4 & $-10,0$ \\
South Atlantic & 327000 & 13,5 & 275000 & 12,7 & $-15,9$ \\
East South Central & 312000 & 12,8 & 285000 & 13,2 & $-8,6$ \\
West South Central & 357000 & 14,7 & 307000 & 14,2 & $-14,0$ \\
Mountain & 123000 & 5,1 & 119000 & 5,5 & $-3,2$ \\
Pacific (ohne Alaska & 154000 & 6,3 & 153000 & 7,0 & $-0,01$ \\
$\quad$ und Hawaii) & & & & & \\
\hline
\end{tabular}

Quelle: Nach U.S. Bureau of the Census 1989: 628, Tab. 1079 (veränd., eig. Berechnung).

Trotz der hohen Konzentrationsrate der Farmen sind dauerhafte und beträchtliche Ertragssteigerungen festzustellen (vgl. Tabelle B-9). Wie der Ertragsindex in Tabelle B-10 zeigt, ist dieses Wachstum in erster Linie auf Produktivitätssteigerungen und nicht auf die Ausweitung der Anbaufläche zurück- 
zuführen. Weniger als die Hälfte der Landfläche der USA ist je landwirtschaftlich genutzt worden oder gilt als Farmland, davon wurden nur rund ein Drittel als Anbaufläche kultiviert, während der Rest unter bestimmten Marktbedingungen als Anbaufläche verfügbar wäre (Lutrell 1984: 34).

Tabelle B-9: Ertragssteigerung ausgewählter Anbauprodukte 1920-1981a

\begin{tabular}{lccc}
\hline Anbauprodukt & $\begin{array}{c}1920 \\
\text { (pro } \text { acre) }\end{array}$ & $\begin{array}{c}1981 \\
\text { (pro acre) }\end{array}$ & $\begin{array}{c}\text { Steigerung } \\
(1920=100)\end{array}$ \\
\hline Weizen (bushels) & 14,0 & 34,5 & $246 \%$ \\
Mais (bushels) & 26,8 & 109,9 & 410 \\
Sojabohnen & 12,7 & 30,4 & 239 \\
Baumwolle (Pfund) & 154,0 & 546,0 & 355 \\
\hline
\end{tabular}

Quelle: Nach Schultz 1984: 48.

a Einzelne Jahre können "Zufälle" sein, d.h. bes. gute oder schlechte Emten anzeigen. Die hier aufgeführten Jahre sollen nur die Tendenz darstellen.

b Ein acre entspricht 0,41 ha.

c Ein bushel entspricht $36,351$.

Tabelle B-10: US-Farmland, tatsächlich kultiviertes Land (Mio. acres) und Ertragsindex

\begin{tabular}{lccc}
\hline Jahr & Farmland & $\begin{array}{c}\text { Tatsächliche } \\
\text { Anbaufläche }\end{array}$ & $\begin{array}{c}\text { Ertragsindex } \\
(1967=100)\end{array}$ \\
\hline 1910 & 879 & 317 & 56 \\
1930 & 990 & 360 & 53 \\
1950 & 1161 & 336 & 69 \\
1964 & 1110 & 292 & 95 \\
1974 & 1017 & 332 & 104 \\
1980 & 1042 & 341 & 115 \\
Gesamtfläche USA & 2316 & & \\
\hline
\end{tabular}

Quelle: Nach Lutrell 1984: 34.

Produktivitätssteigerungen sind auf den höheren Kapitaleinsatz zurückzuführen, der sich in verstärkter Automation und Anwendung neuester wissenschaftlicher und technologischer Erkenntnisse manifestiert. Zwischen 1950 und 1980 versechsfachte sich der Gebrauch von Agrochemikalien und trug damit wesentlich zur Ertragssteigerung bei, ebenso wie gentechnologische Entwicklungen, die bei einer Vielzahl von Anbaupflanzen (Tomaten, Bohnen, Salat u.a.) die automatisierte Ernte ermöglichten (Clark 1985: 148). Der wachsende Kapitaleinsatz in der Landwirtschaft ist von einem Ansteigen der durchschnittlichen Farmngrößen und der Ausbildung einer dualen Struktur begleitet, d.h. einem relativ kleinen Prozentsatz sehr großer "Megafarmen", die vertikalintegrierten Untemehmen angeschlossen sind, und einen großen Anteil von kleinen, oft marginalwirtschaftenden Farmen (Windhorst 1987: 474f.). Zwi- 
schen 1940 und 1980 stieg die Durchschnittsgröße der Farmen von 67,5 auf 173,7 ha (167 bzw. 430 acres) durch Erwerb von Ländereien aufgegebener Farmen. Dieser Trend setzte sich in jüngerer Zeit in allen Regionen fort (vgl. Tabelle B-11).

Tabelle B-11: Veränderung in der Durchschnittsgröße der Farmen (in acres) $1980-1988^{\mathrm{a}}$

\begin{tabular}{lrrrr}
\hline & \multicolumn{2}{c}{1980} & \multicolumn{2}{c}{1988} \\
& Min. & Max. & Min. & Max. \\
\hline New England & 87 & 226 & 96 & 223 \\
Middle Atlantic & 109 & 200 & 112 & 215 \\
East North Central & 171 & 269 & 186 & 345 \\
West North Central & 261 & 1169 & 9 & 1278 \\
South Atlantic & 126 & 344 & 150 & 325 \\
East South Central & 142 & 265 & 136 & 314 \\
West South Central & 273 & 731 & 279 & 846 \\
Mountain & 623 & 5080 & 609 & 4506 \\
Pacific (ohne Alaska & 417 & 517 & 411 & 488 \\
$\quad$ und Hawaii) & & & & \\
\hline
\end{tabular}

Quelle: Nach U.S. Bureau of the Census 1989: 628, Tab. 1079 (veränd.).

a Die hier angegebenen Werte sind die höchsten bzw. niedrigsten state-level-Durchschnittsgrößen innerhalb der Region.

Tabelle B-12: Farmklassen und ihr Anteil am Gesamtfarmeinkommen in den USA 1985

\begin{tabular}{ccc}
\hline $\begin{array}{l}\text { Farmklassen } \\
\text { (Marktabsatz } \\
\text { in Tsd. \$) }\end{array}$ & $\begin{array}{l}\text { \% des Brutto- } \\
\text { Farmeinkommens }\end{array}$ & \% der Farmen \\
\hline unter 19999 & 9,4 & \\
$20000-99999$ & 19,3 & 63,5 \\
$100000-249999$ & 22,2 & 22,8 \\
$250000-499999$ & 16,9 \\
500000 und mehr & 32,2 & 9,3 \\
USA gesamt & 167,7 Mrd. \$ & $3,2\} 4,5 \%$ \\
\hline
\end{tabular}

Quelle: U.S. Bureau of the Census 1989: 635, Tab. 1098 (veränd., eig. Berechn.). 
Die Unterschiede zwischen den Regionen reflektieren allerdings in erster Linie agrarökologische und historische Entwicklungen (wie z.B. größere Landzuteilungen durch die Heimstättengesetzgebung von 1862 in ariden Gebieten westlich des 100. Längengrades) und weniger den postindustriellen Wandel zu einer dualen Struktur. Dieser Wandel ist in den Größenveränderungen innerhalb der jeweiligen Region angedeutet und für die USA als Ganzes in Tabelle B-12 dargelegt. Demnach hat eine große Anzahl von Farmen nur einen geringen Anteil an der Agrarproduktion, und ein kleiner Prozentsatz großer Farmen vereint den überwiegenden Teil der Erzeugung und des Farmeinkommens auf sich (Windhorst 1987: 474). Der Rückgang der Klein- und Kleinstfarmen wird als direkte Voraussetzung angesehen, um die Leistungsfähigkeit des Farmsektors zu steigern, wobei der Größenmaßstab der Farmen von besonderer Bedeutung ist (Birdsall/Florin 1981: 283-287).

In den Regionen mit hohem Anteil an Kleinfarmen haben sich im Trend zur automatisierten, rentableren Großfarm die genossenschaftliche Organisation und die Vergrößerung durch Miete und Pacht von Land und Wirtschaftsgebäuden aufgegebener Farmen durchgesetzt (Windhorst 1987). Die Farmergenossenschaften mit 4,8 Mio. Mitgliedern 1985 entstanden

... als ein Korrektiv gegenüber der Konzentration der ökonomischen Macht und als Anwalt bzw. Selbsthilfeorganisation für die Schwachen im ökonomischen Wettbewerb (Klohn 1987: 36 und Klohn 1990).

Die regionale Verteilung der Genossenschaftsmitglieder zeigt Schwerpunkte in den Regionen East und West North Central, Middle und South Atlantic und East South Central (vgl. Schaubild B-9 und Klohn 1987: 43).

Farmen, die sich durch Anmietung aufgegebener Betriebe vergrößern, sind oft stark fragmentiert, wobei Distanzen von 8 bis 25 Meilen zu einzelnen Parzellen keine Seltenheit sind. Die weite Streuung der Felder ist in vielen Fällen angestrebt, um bei schweren Unwettern nicht die gesamte Ernte einbüßen zu müssen. Dabei wird der Farmer oft zum suitcase farmer oder sidewalk farmer, der von seinem Wohnsitz in der nahe gelegenen Kleinstadt zu seinen verschiedenen Arbeitsplätzen pendelt. Suitcase farming ist vor allem in den Weizenanbaugebieten von Dakota bis Texas verbreitet (Smith 1975 und Birdsall/Florin 1981: 289). Trotz solcher Betriebsvergrößerungen, die eine stärkere Automatisierung erlauben und teilweise erhebliche Steigerungen des Marktwerts der Farmen mit sich brachten, verlief das Farmsterben insgesamt von 1982 bis 1987 mit einer Abnahme von 2,241 Mio. auf 2,088 Mio. Farmen schneller als z.B. zwischen 1974 und 1982 mit einer Abnahme von 2,314 Mio. auf 2,241 Mio., wobei die traditionellen Farmstaaten besonders betroffen waren (U.S. Bureau of the Census 1990: 639, Tabellen 1102 und 1108; McKinzie/Baker/Tyner 1987). 


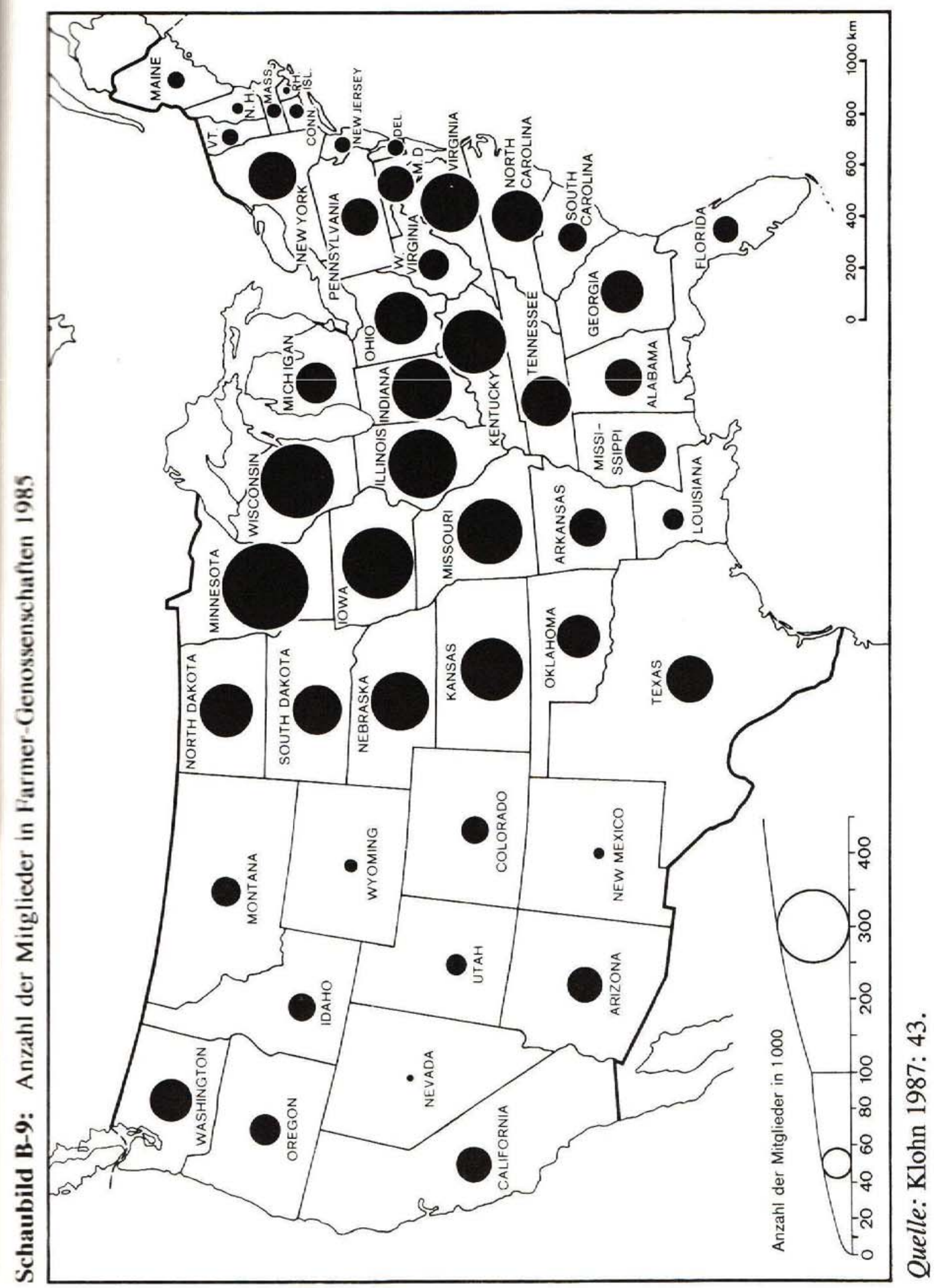


Obwohl auf bundes- und agrarpolitischer Ebene die Farmkrise einerseits als Gesundschrumpfung des Farmsektors begrüßt wird und einige Unterstützungsprogramme ausliefen oder drastisch gekürzt wurden, bleiben einige Förderungsmaßnahmen bestehen (oft wegen der wahlpolitischen Bedeutung der Farmregionen). Dazu gehören Ausgleichszahlungen, die die Bundesregierung dann gewährt, wenn die Preise für Agrarprodukte unter ein festgesetztes Preisniveau fallen (Windhorst 1986).

Tabelle B-13: Durchschnittswert (in \$) von Nutzland und Wirtschaftsgebäuden 1984, 1986 und 1988 pro acre

\begin{tabular}{lrrr}
\hline & 1984 & 1986 & 1988 \\
\hline New England & 1785 & 2360 & 3218 \\
Middle Atlantic & 1906 & 2062 & 2988 \\
East North Central & 1421 & 972 & 914 \\
West North Central & 77 & 477 & 462 \\
South Atlantic & 1332 & 1198 & 1249 \\
East South Central & 950 & 844 & 819 \\
West South Central & 854 & 683 & 560 \\
Mountain & 381 & 300 & 278 \\
Pacific (ohne Alaska & 1192 & 968 & 853 \\
$\quad$ und Hawaii) & & & \\
\hline
\end{tabular}

Quelle: Nach U.S. Bureau of the Census 1987: 626, Tab. 1110 und 1989: 631, Tab. 1088.

1985 machten die staatlichen Ausgleichszahlungen an Farmer 5\% des gesamten Farmeinkommens aus, in den von der Farmkrise am stärksten betroffenen Regionen West und East North Central waren es rund 8\%, in Mountain und West South Central sogar 16\% und 22\% (U.S. Bureau of the Census 1987: $633)$.

Während Miet- und Pachtarrangements im Mittelwesten zur Vergrößerung der landwirtschaftlichen Nutzfläche und damit dem besseren Kapitaleinsatz dienen, versuchen Farmen im Einzugsbereich städtischer Verdichtungsräume eher durch Änderung der Betriebsorganisation die Vorteile von Großfarmen zu erhalten. Zum einen lassen sich viele Familienfarmen als selbständige corporations eintragen, um die Kreditfähigkeit und Möglichkeiten steuerlicher Abschreibung zu steigern. Zum anderen werden viele Familienfarmen Vertragspartner (subcontractors) und Zulieferbetriebe für vertikalintegrierte Agrarkonzerne, die vom Saatgut bis zum Supermarkt das gesamte Lebensmittelangebot erstellen (Windhorst 1987; Clark 1985: 151). Der Anteil der corporate farms an allen Farmen betrug 1987 3,2\%, wovon 86,7\% noch Familienbetriebe und $9,6 \%$ den vertikalintegrierten Unternehmen angeschlossen waren. Corporate 


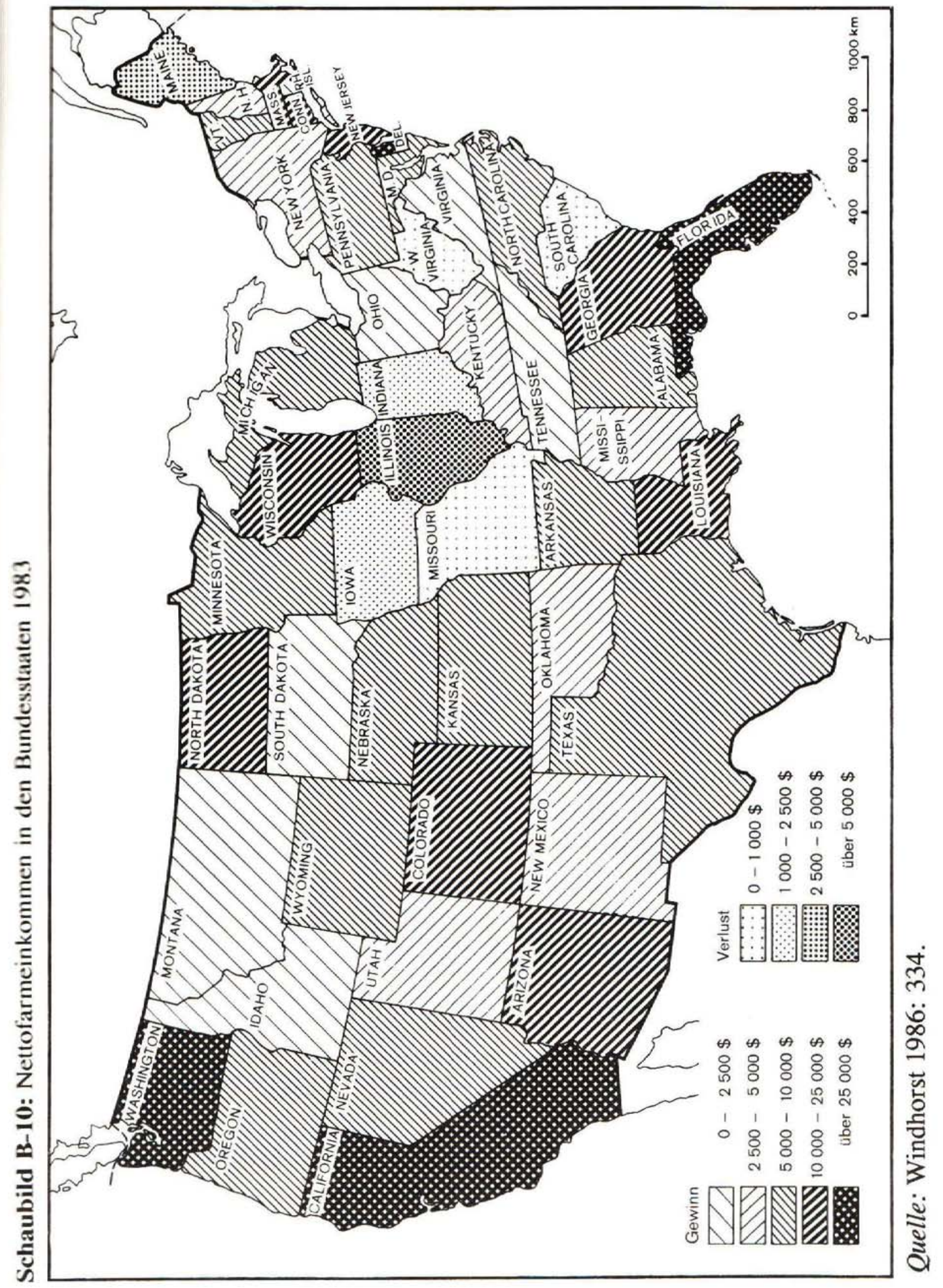


farms besaßen 1987 11,4\% der gesamten landwirtschaftlichen Nutzflächen und Wirtschaftsgebäude der USA und erzeugten $25,6 \%$ des Verkaufswertes der USAgrarproduktion (U.S. Bureau of the Census 1990: 641, Tabelle 1107). Corporate farms sind oft produktkonzentriert, z.B. bei Geflügel, Eiern, Gemüse, Zitrusfrüchten und Rindfleisch. Daher dominieren sie auch in einigen geographischen Regionen. Schon 1969 lieferten Corporate farms über die Hälfte der kalifornischen Agrarprodukte, in einigen ausgewählten Gemüse- und Obstsorten sogar 90\% (Parsons 1977; Clark 1985: 152). Agroindustrielle Unternehmen stellen seit Beginn der 80er Jahre auch $60 \%$ der US-Rindfleischproduktion, wobei Texas und Kalifornien Hauptstandorte sind. Auch die Geflügelproduktion, die größtenteils in fabrikähnlichen, fließbandbetriebenen Anlagen oder in kleineren Vertragsfarmen betrieben wird, ist geographisch konzentriert, und zwar halbkreisförmig um die "Megalopolis" Boston-Washington und die Großstädte an der Westküste herum sowie im Alten Süden (Windhorst 1987).

Tabelle B-14: Agrarprodukte mit dem höchsten Verkaufserlös 1985a

\begin{tabular}{|l|l|}
\hline New England & $\begin{array}{l}\text { Milch und Molkereiprodukte, Rindfleisch, Treib- } \\
\text { hauskulturen, Kartoffeln, Äpfel }\end{array}$ \\
\hline Middle Atlantic & $\begin{array}{l}\text { Milch und Molkereiprodukte, Rindfleisch, Eier, } \\
\text { Treibhaus- und Sonderkulturen }\end{array}$ \\
\hline East North Central & $\begin{array}{l}\text { Mais, Sojabohnen, Milch und Molkereiprodukte, } \\
\text { Rind- und Schweinefleisch }\end{array}$ \\
\hline West North Central & $\begin{array}{l}\text { Rindfleisch, Milch und Molkereiprodukte, Schweine- } \\
\text { fleisch, Mais, Weizen, Roggen, Hirse }\end{array}$ \\
\hline South Atlantic & $\begin{array}{l}\text { Geflügel, Rindfleisch, Milch und Molkereiprodukte, } \\
\text { Mais, Sojabohnen, Schweinefleisch, Tabak, Eier, } \\
\text { Erdnüsse, Zitrusfrüchte, Treibhaus- und Sonderkul- } \\
\text { turen }\end{array}$ \\
\hline East South Central & $\begin{array}{l}\text { Rindfleisch, Tabak, Geflügel, Sojabohnen, Milch } \\
\text { und Molkereiprodukte }\end{array}$ \\
\hline West South Central & $\begin{array}{l}\text { Rindfleisch, Geflügel, Sojabohnen, Reis, Baumwol- } \\
\text { le, Weizen }\end{array}$ \\
\hline Mountain & $\begin{array}{l}\text { Rindfleisch, Weizen, Kartoffeln, Milch und Molke- } \\
\text { reiprodukte, Baumwolle, Schafe, Gerste, Mais }\end{array}$ \\
\hline $\begin{array}{l}\text { Pacific (ohne } \\
\text { Alaska und Hawaii) }\end{array}$ & $\begin{array}{l}\text { Weizen, Milch und Molkereiprodukte, Rindfleisch, } \\
\text { Treibhaus- und Sonderkulturen }\end{array}$ \\
\hline
\end{tabular}

Quelle: Nach U.S. Bureau of the Census 1987: 633, Tab. 1125.

a Zusammengestellt aufgrund der drei führenden Produkte in den einzelnen Bundesstaaten der jeweiligen Region. 
Die regionalen Schwerpunkte in der Erzeugung anderer Produkte sind aus Tabelle B-14 zu entnehmen. Insgesamt ergibt sich, den Thünenschen Intensitătskreisen nicht unähnlich, eine halbkreisförmige Einteilung in Produktzonen am städtische Ballungszentren. Die sehr profitablen, aber leicht verderblichen Molkereiprodukte, Treibhauskulturen, Obst-, Geflügel- und Eierproduktionen befinden sich im unmittelbaren Hinterland der Großstädte. Die Produktion erstreckt sich insgesamt um die südlichen Großen Seen, um den Verdichtungsraum Boston-Washington und die kaliformischen Städte herum. Viehhaltung und gemischter Pflanzenbau (bei Mais, Sojabohnen und Hirse zum großen Teil für die Viehmast) befinden sich weiter entfernt in den Regionen East und West North Central, East und West South Central und Mountain. Welche Produkte in den einzelnen Bundesstaaten in der Agrarproduktion führend sind, ist aus Schaubild B-10 ersichtlich.

Eine genaue Betrachtung der relativ spezialisierten Agrarregionen zeigt, daß die Anwendung des traditionellen Belt-Konzeptes für die amerikanische Landwirtschaft keine Gültigkeit mehr besitzt. Seit dem ersten klassischen Modell nach Baker (1926) sowie Haystead und Fite (1963, vgl. Schaubild B-12) sind regionale Schwerpunktverlagerungen eingetreten.

Dazu gehören z.B. die Ausdehnung des Hybridenmaisanbaus in den Norden, eine Verdrängung des Maisanbaus im ehemaligen Corn Belt durch die ertragreichere und profitablere Sojabohne, die eine breite Anwendung in der Milch- und Fleischproduktion, der Viehmast, Herstellung von Plastik und Farben und in anderen industriellen Produktionsprozessen hat (Clark 1985: 169). Ferner erfolgten eine Ausdehnung des Hirseanbaus in den Weizengürtel, der Rindermast in den Süden, nach Kalifornien und in den Nordosten, hervorgerufen durch steigende Rindfleischpreise auf dem Binnenmarkt und in den Handelspartnerländern der USA (z.B. Japan). Die ursprünglich im Mittelwesten gelegene Geflügelhaltung verlagerte sich in den Süden sowie an die atlantische Küste und der Baumwollanbau vom Alten Süden nach Texas, Arizona, New Mexico und Kaliformien (Windhorst 1986).

Im Gegensatz zum althergebrachten Beltkonzept, das die Ausbildung von Anbaugürteln aufgrund von Klima, Vegetations- und Bodenzonen nachzeichnet, reflektieren die postindustriellen Agrarräume oder Schwerpunktgebiete nur noch teilweise agrarökologische Bedingungen und in nicht unbedeutender Weise staatliche Intervention. Umfassende staatliche Maßnahmen (z.B. Bodenberatungsdienst, Anbaubeschränkungen durch den Agricultural Adjustment Act I von 1933, Preisgarantien durch den Agricultural Adjustment Act Il von 1953, Eindämmung der Erosionsanfälligkeit und staatlich geförderte Diversifikation der Farmproduktion durch den Agricultural Stabilization Act von 1965, das Emergency Feed Grain Program von 1961 und das Cropland Adjustment Program von 1966 und andere Maßnahmen) führten zur Verschiebung von Anbauarealen bestimmter Pflanzen oder von Mastgebieten für Vieh (Blume 1987, Bd. 1). Vor 
Schaubild B-11: Agrarprodukte mit den höchsten Anteilen am Gesamtwert der Agrarproduktion 1985

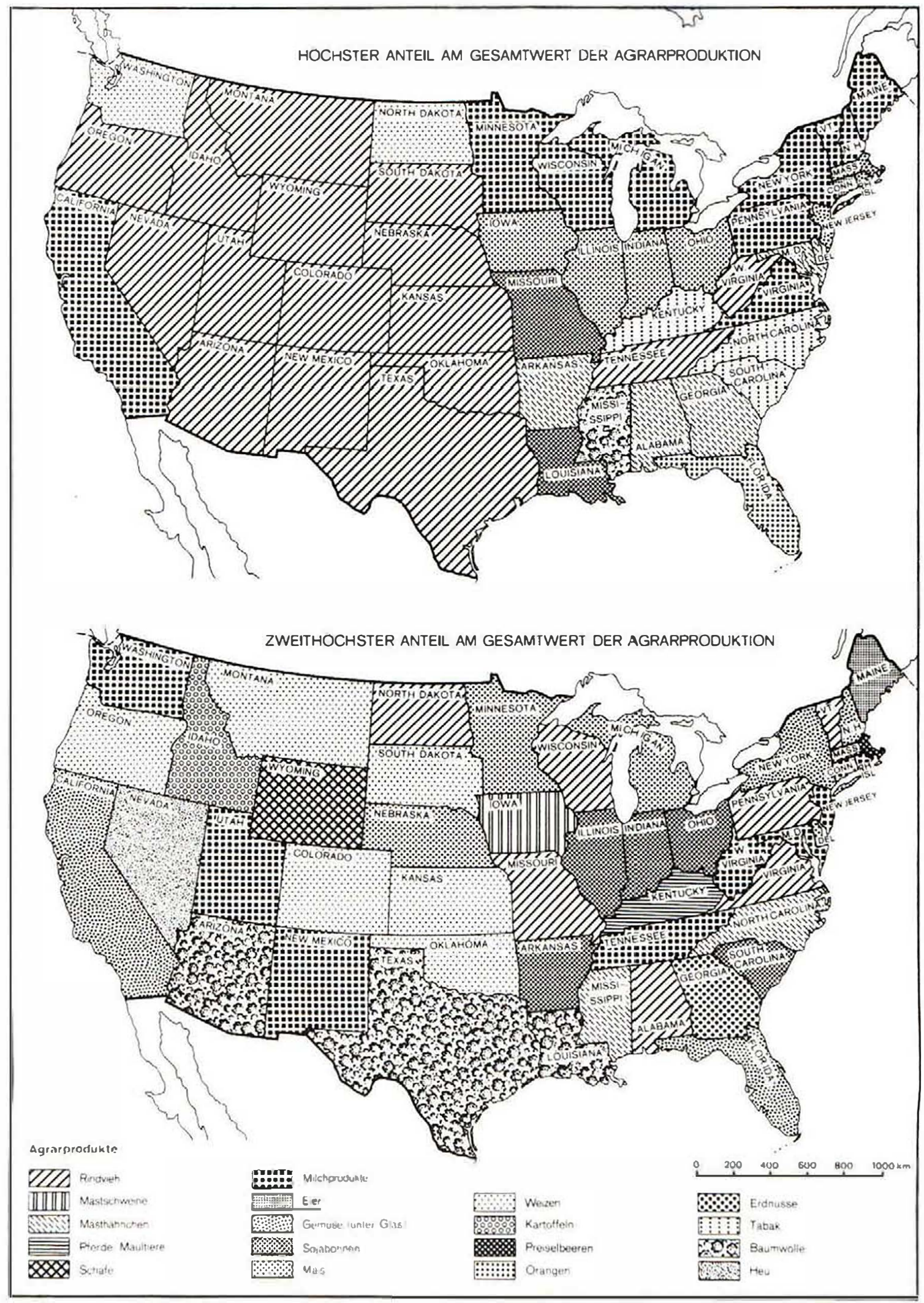

Quelle: U.S. Bureau of the Census 1987: 633, Tab. 1125. 
allem die Maßnahmen von 1961 und 1966 schufen finanzielle Anreize, Maisund Hirseanbauareale zugunsten anderer Produkte zu reduzieren oder das so freigewordene Land in einer staatlichen Bodenbank für handelspolitische Zwecke verfügbar zu machen (Estall 1980). Der direkte oder indirekte Effekt eines anderen Gesetzes, des Public Law 480, war z.B., den Anbau von Weizen und die Ausdehnung des Weizenanbauareals zu fördern, nachdem die Farmer für ihren "P.L. 480 Weizen" seit Mitte der 50er Jahre einen gesicherten Absatzmarkt in Ländern der Dritten Welt erhielten. Ähnliche Auswirkungen hatten verstärkte Handelsbeziehungen mit der UdSSR seit Beginn der 70er Jahre.

Bis auf das mediterran-klimatische Kalifornien, das subtropische Florida und den ariden Westen - Regionen, deren Landwirtschaft sehr stark klimaabhängig ist - stellen die postindustriellen Agrarzonen nicht mehr nur agrarökologische Gegebenheiten, sondern eine durch staatliche Eingriffe beeinflußte Landwirtschaft dar. Zumindest in den Übergangszonen agrarökologischer Anbaugürtel wird die tatsächliche Nutzung oft durch eine Kombination von Technik, Kommunikation und Politik bestimmt. Mit Hilfe der Erdfernerkundung kann das amerikanische Landwirtschaftsministerium Anbau, Emtevolumen, Ausmaß des Schädlings- oder Krankheitsbefalls in anderen Ländern cinschließlich der Entwicklungsländer genauestens verfolgen und eingrenzen. Nur wenige Tage nach der Aussaat von Getreide in der UdSSR z.B. kann man mit Hilfe der Satellitendaten ermitteln, ob und in welcher Höhe sich Fehlschläge abzeichnen. Dem kann man durch einen sehr effektiven, staatlichen landwirtschaftlichen Beratungsdienst (viele Megafarmen haben ihren eigenen inhouse-Berater des Agricultural Extension Service) zum Teil noch in derselben Anbausaison durch verstärkten Anbau von Getreide in einigen Agrarstaaten der USA begegnen. Die heutigen technischen Möglichkeiten verleiten zu der provokativen Aussage, daß eine Übereinstimmung der gegenwärtigen mit den klassischen (klima- und bodenabhängigen) Anbauarealen eher zufällig ist. Auf jeden Fall können sich Anbaugürtel mit neuen Preissignalen und Subventionen aus Washington sowie mit der jeweiligen Außenhandelspolitik ändern. Während die Landwirtschaft niemals footloose sein kann wie verschiedene Sekundär- und Tertiärbereiche, ist sie doch im hohen Maße anpassungsfähig geworden, wobei die jeweilige geographische Anbauzone nicht mehr nur vom physischen, sondern auch stark vom politischen Klima geprägt ist.

Einige Anbaugebiete haben sich als besonders anpassungsfähig erwiesen und gewinnen daher gesamtstaatlich an Bedeutung. Tabelle B-15 zeigt die Bedeutung einzelner Regionen in der Agrarproduktion. Der Mittelwesten (East und West North Central) ist mit 41,2\% Anteil am Gesamtverkaufserlös der Agrarproduktion noch die wichtigste Anbauregion der USA, der Süden (vor allem East und West South Central) und der Westen (Mountain und Pacific) folgen mit $18,0 \%$ bzw. $22,6 \%$. 


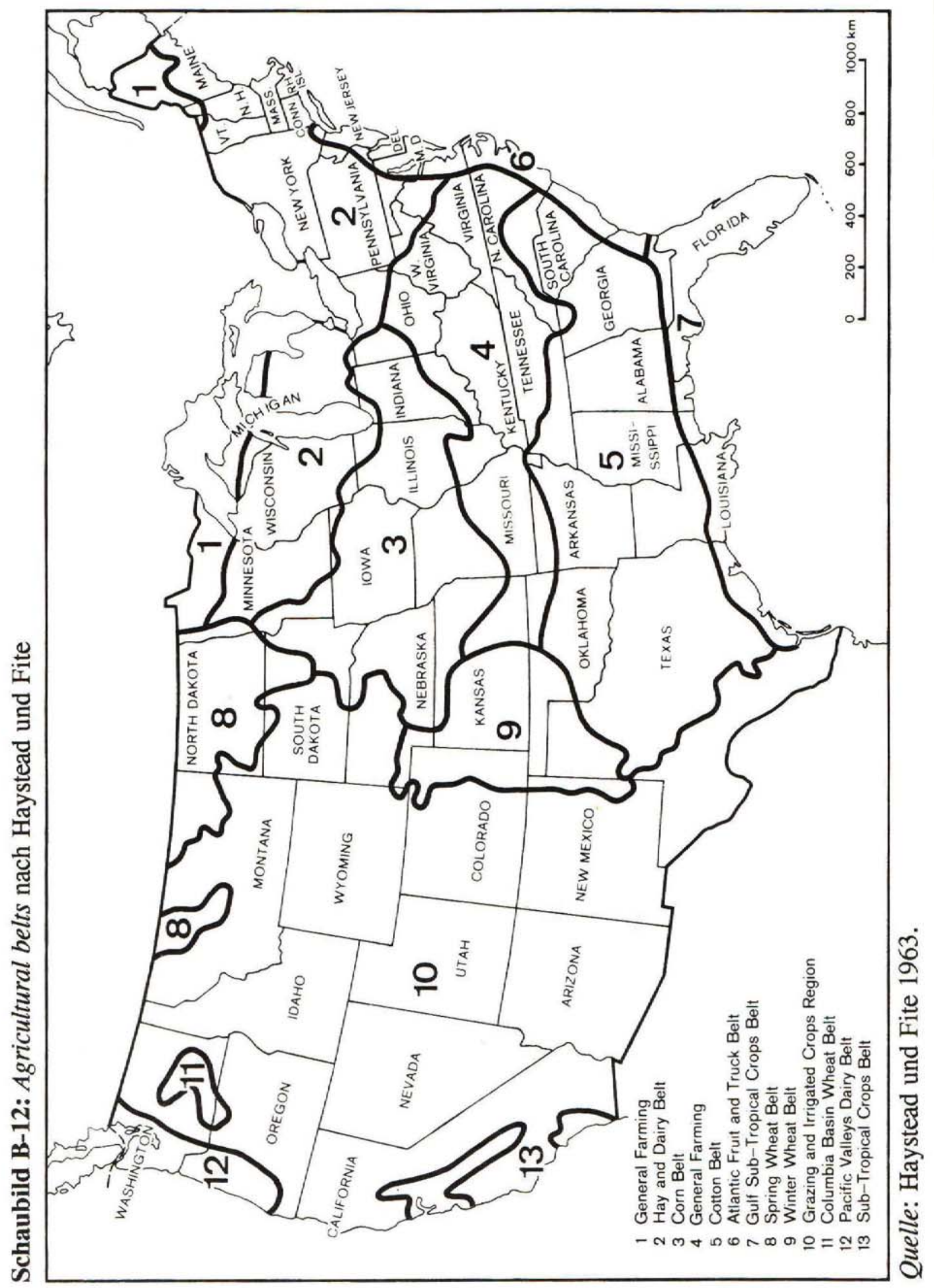


Tabelle B-15: Anteil am Verkaufserlös für Agrarprodukte 1987

\begin{tabular}{lrlr}
\hline Region & $\%$ & Führende & $\%$ \\
& & Bundesstaaten & \\
New England & 1,3 & Kalifornien & 11,2 \\
Middle Atlantic & 4,6 & Texas & 6,6 \\
East North Central & 15,2 & Iowa & 6,4 \\
West North Central & 26,0 & Illinois & 4,5 \\
South Atlantic & 11,9 & Nebraska & 4,9 \\
East South Central & 6,1 & Minnesota & 4,2 \\
West South Central & 11,9 & Kansas & 4,1 \\
Mountain & 8,0 & Wisconsin & 3,6 \\
Pacific (ohne Alaska & 14,6 & Florida & 3,8 \\
$\quad$ und Hawaii) & & Indiana & 2,8 \\
& & Ohio & 2,5 \\
& & zusammen & 59,6 \\
\hline
\end{tabular}

Quelle: Nach U.S. Bureau of the Census 1989: 637, Tab. 1100 (veränd., eig. Berechn.).

Eine Betrachtung der führenden Bundesstaaten zeigt jedoch eine ähnliche postindustrielle Entwicklung, wie sie auch im Industriesektor zu beobachten war: ein relativer Bedeutungsverlust der "altagraren" Produktionsgebiete wie Ohio, Indiana, Wisconsin, Kansas, Nebraska und Illinois bei gleichzeitigem Aufschwung "neuagrarer" Kernländer wie Texas und Kalifornien, die sich im Gegensatz zur einseitigen Produktionsausrichtung altagrarer Regionen durch eine Vielzahl von teuren Spezialkulturen auszeichnen, wie z.B. Früchte, Nüsse, Gemüse, Wein und Obst. Beachtenswert bei den neuagraren Gebieten, die in der postindustriellen Phase zu den wichtigsten Anbauregionen wurden, ist nicht nur die Kombination der angebauten Produkte. Diese wurden z.T. schon Ende des 19. Jahrhunderts in regionalen Studien der USA registriert und später zum subtropical crop belt zusammengefaßt. Auffallend ist vielmehr der hohe Anteil, den diese Produkte am Verkaufserlös haben. Dies spiegelt sicher auch die Präferenz der amerikanischen Konsumenten wider, die in den letzten Jahrzehnten mit steigendem Einkommen weniger Nachfrage nach Grundnahrungsmitteln, sondern mehr nach Nahrungsmitteln gehobener Preisklassen zeigten. Der relative Bedeutungsverlust altagrarer Regionen ist ebenso damit in Verbindung zu bringen wie mit den schon erwähnten Faktoren (Preisentwicklung auf dem Weltmarkt, finanzielle Überlastung, Mißernten, Limitierung staatlicher Subventionen). Da das alte landwirtschaftliche Kernland als Kornkammer der USA stark exportorientiert ist, kann es je nach Außen- und Handelspolitik wieder stark an Bedeutung gewinnen:

... agricultural products increased in strategic importance as President Carter's grain embargo of 1979 emphasized. The prosperity of 
American agriculture is likely to be determined by foreign policy in the future as it was by price supports and acreage control provisions in the past (Clark 1985: 157).

\section{Zusammenfassung}

Die im Industriezeitalter entstandene regionale Struktur der US-amerikanischen Wirtschaft unterliegt seit Mitte des 20. Jahrhunderts verstärkt einem Wandel, und zwar findet eine Ablösung des alten zentral-peripheren Gefüges durch ein Muster mit mehreren Wirtschaftszentren statt, das bedeutet postindustrielles Wirtschaftswachstum in ehemals strukturschwachen peripheren Räumen sowie Funktionseinbußen des altindustrialisierten und altagraren Kernlandes. Der starke Aufschwung des tertiären Sektors, soziale Entwicklungen und Änderungen im demographischen Verhalten, Fortschritte im Kommunikationswesen und andere technische Neuerungen sowie die Wirtschafts- und Strukturpolitik der öffentlichen Hand sind einige der Ursachen. Bundes- und einzelstaatliche Maßnahmen, wie z.B. Investitionen im Transportsektor ebneten der Industrialisierung peripherer Räume den Weg, Vertragsvergabe an Hochtechnologieindustrien und -dienstleistungsbetriebe begünstigten neue Standortschwerpunkte, agrarpolitische Maßnahmen beeinflußten Agrarproduktion und Anbauareale.

Nicht selten haben diese Veränderungen zu Krisen geführt, die alt- und neuindustrialisierte Gebiete, klassische und neue Standortschwerpunkte des Agrarsektors, sowie die tertiärwirtschaftlich bedeutenden städtischen Ballungszentren und auch die Hochtechnologieenklaven betreffen. Diese Krisen ihrerseits haben zu weiteren bundes- und einzelstaatlichen Maßnahmen geführt, die unterschiedlichen Einflu $\beta$ auf das regionale Wirtschaftswachstum haben. Insgesamt läßt sich daher sagen, daß bundes- und einzelstaatliche Maßnahmen die wirtschaftliche Entwicklung in den einzelnen Regionen in entscheidender Weise geprägt haben und als ein Leitmotiv im Wandel der wirtschaftsgeographischen Struktur anzusehen sind. 